\title{
Matrimonio forzado y embarazo adolescente en indígenas en Amatenango del Valle, Chiapas. Una mirada desde las relaciones de género y el cambio reproductivo
}

\author{
Forced marriage and adolescent pregnancy in indigenous people \\ in Amatenango del Valle, Chiapas. An approximation from gender \\ relationes and reproductive change
}

\author{
Juana Luna-Pérez, Austreberta Nazar-Beutelspacher, Ramón \\ Mariaca-Méndez y Dulce Karol Ramírez-López \\ Consultora Independiente, Departamento de Salud de El Colegio de la Frontera \\ Sur en Chiapas, México, Director General de Estadistica e Información Ambien- \\ tal de la SEMARNAT, México, Ciencias Políticas y Administración Pública de la \\ Universidad de Ciencias y Artes de Chiapas, México
}

Resumen

En este estudio, se analizan los cambios en la frecuencia del matrimonio forzado y del embarazo adolescente en una comunidad indígena tseltal de Chiapas. Fueron utilizadas metodologías cuantitativas y cualitativas, así como una revisión de fuentes secundarias de información. Los resultados muestran que el matrimonio forzado ha disminuido y, entre las más jóvenes se han incrementado las uniones "voluntarias" que acompañan la disminución de la edad de unión. Lo anterior, pese al incremento de la escolaridad de las mujeres, el acceso al trabajo remunerado y a los métodos anticonceptivos. Se discute el papel de las relaciones de género en el matrimonio forzado y el embarazo adolescente, así como el posible impacto que tendría la modificación de la legislación para prohibir el matrimonio infantil en México.

Palabras clave: Matrimonio infantil, Chiapas, trabajo remunerado, escolaridad, anticoncepción.

Abstract

In this study, the changes in the frequency of forced marriage and adolescent pregnancy in an indigenous Tseltal community of Chiapas are analyzed. Quantitative and qualitative methodologies were used, as well as a review of secondary sources of information. The results show that forced marriage has decreased and, among the youngest, the "voluntary" unions that accompany the decrease in the age of union have increased. The foregoing, despite the increase in the education of women, access to paid work and contraceptive methods. The role of gender relations in forced marriage and adolescent pregnancy is discussed, as well as the possible impact that the modification of the legislation to prohibit child marriage in Mexico would have.

Keywords: Child marriage, Chiapas, paid work, schooling, contraception. 


\section{INTRODUCCIÓN}

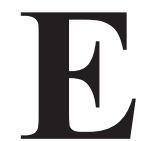

1 embarazo adolescente y el matrimonio forzado son dos temas relacionados que han adquirido importancia por sus implicaciones negativas en la salud y calidad de vida de los y las menores, a la vez que se enmarcan en las discusiones de protección a la infancia y de sus derechos humanos. Ambos fenómenos ocurren principalmente en países del tercer mundo, en los sectores de población más pobres y marginados y, en el caso de América Latina, en las poblaciones indígenas y rurales. Constituyen, asimismo, un tema central en las discusiones recientes sobre el desarrollo (UNFPA, 2012).

El matrimonio forzado es una práctica muy antigua que en los últimos años ha sido objeto de gran atención académica, política y social, particularmente en su dimensión de expresión de violencia contra la niñez, y de violación de los derechos humanos de niños, niñas y adolescentes (UNICEF, 2014; Warria, 2017; Frías, 2017). Suele identificarse con la participación en la decisión por parte de mujeres y hombres, principalmente de las mujeres, y suele asumirse que ocurre en menores de edad.

El matrimonio forzado es "todo aquel que se celebra sin el consentimiento pleno y libre de al menos uno de los contrayentes, debido a coacciones o a una intensa presión social o familiar" (UNICEF, 2014: 4). El matrimonio forzado es una práctica aún común en cientos de países (UNFPA, 2012). El Fondo de Población de las Naciones Unidas (2012), estima que, con excepción de China, una de cada tres niñas de países en desarrollo contraerá matrimonio antes de cumplir 18 años, y que una de cada nueve menores lo hará antes de los 15 años. Lo anterior ocurre pese a que en el ámbito internacional existe acuerdo casi generalizado ${ }^{1}$ de que el matrimonio forzado es una violación a los derechos humanos de las menores, por lo que deben generarse acciones globales para erradicarlo y mejorar sus condiciones de vida (Machel, Pires y Carlsson, 2013; Simmons y Burn, 2013; Frulli, 2008).

Aunque el matrimonio forzado se ha reportado en varones y mujeres adolescentes y adultos/adultas jóvenes, generalmente ocurre en niñas y mujeres adolescentes (antes de cumplir 18 años), bajo la coerción e incluso violencia por parte de los padres y de la sociedad (UNICEF, 2014). La UNICEF (2014) afirma que los y las menores no pueden consentir de manera voluntaria sobre su vida futura, debido a la inmadurez psicológica, fí-

1 Frías (2017), citando a Chantler, K. (2012), señala que no siempre se reconoce el matrimonio forzado como una forma de violencia. 
sica y emocional que ellos y ellas presentan. La falta de poder de decisión y de la presión social, la ausencia de otras opciones de vida mediadas por las normas culturales arraigadas en las comunidades, y las condiciones de pobreza, marginación y desigualdad de género, son algunos de los elementos contextuales que subyacen a la "voluntad" de las menores de edad para establecer uniones tempranas y embarazos precoces, particularmente en las poblaciones indígenas (Menkes y Suárez, 2003; Stern, 2004; Arias et al., 2008). La diferencia con el concepto de "matrimonio forzado", tal como ha sido definido por UNICEF (2014), estriba en que la unión "voluntaria" es reconocida por las mujeres como un acto en el que ellas consienten la unión; sin embargo, en las menores de edad, el matrimonio "voluntario" no puede ser interpretado como un acto pleno e informado y sujeto a coerción o presiones sociales (UNFPA, 2013), debido a que por edad aún no tienen la capacidad de tomar decisiones sobre su vida reproductiva; su "decisión" de aceptar la unión es resultado de su escaso poder de decisión y de la presión social de su entorno, además de la falta de otras opciones de vida.

El matrimonio forzado se ha considerado una expresión más de violencia contra menores que puede ser entendida como tráfico de niños y niñas (Warria, 2017), el cual puede ocurrir en situaciones que incluyen la violación, el rapto, el matrimonio con el violador, y otras expresiones de violencia y coerción por parte de los familiares de la niña para lograr el matrimonio (Kopelman, 2016; Frías, 2017). Asimismo, ha sido reconocido como un problema social y de salud pública, principalmente en la salud sexual y reproductiva de las niñas y mujeres, debido a que experimentan un inicio de relaciones sexuales de manera forzada, la probabilidad de contraer infecciones de transmisión sexual, están expuestas a sufrir violencia sexual y de género, con los consecuentes daños a la salud mental, pero de manera importante se exponen al embarazo temprano que incrementa los riesgos de morbilidad y mortalidad materno-infantil (Organización Mundial de la Salud, 2012).

Desde la mirada de género, el matrimonio forzado es el resultado del ejercicio abusivo del poder en el que, a la desventaja del sexo, se agrega la desventaja por ser menor de edad, debido a que generalmente ocurre con mujeres en la niñez o adolescencia bajo condiciones sociales en las que se asume que nacen y crecen para casarse y tener hijos como el único rol y función reconocida para la mayoría de las sociedades (UNFPA, 2012). El embarazo adolescente y el matrimonio forzado suelen compartir esas condiciones sociales y están articulados entre sí. La condición de desventaja social de las mujeres es un aspecto clave de la reproducción y control so- 
cial (UNFPA, 2012; Scott et al., 2014). Así los aspectos institucionales de las relaciones de género ejercen una influencia decisiva en los arreglos que conllevan a la reproducción de la desigualdad en la familia y la comunidad (Sen, 1990), el matrimonio de menores de edad —más aún si es forzado-, da cuenta de esos arreglos. No se trata de decisiones individuales sino de formas comunitarias de relación. Los arreglos matrimoniales ocurren en los contextos donde existe control social y familiar sobre la vida de hombres y de mujeres, y donde las mujeres entran al mercado matrimonial desde la pubertad. En el matrimonio forzado, la mayoría de las veces las parejas no se conocen antes de casarse, normalmente las mujeres son pedidas por el varón a los padres, y el matrimonio es concertado, independientemente de la "voluntad" de las niñas o adolescentes.

Entre los factores que contribuyen a las uniones tempranas y forzados en las zonas pobres, muchas de ellas indígenas (en el caso de América Latina), o de algunos grupos religiosos, está el de "asegurar la subsistencia económica de las niñas y las mujeres que no tienen acceso a recursos productivos y que viven en situaciones de extrema pobreza" (UNICEF, 2014: 3 ), puesto que casar a las niñas puede traer ventajas económicas, en que los padres pueden acordar la unión de las hijas a cambio de dinero o de otros bienes. No obstante, las prácticas de matrimonio infantil también se presentan en algunos países con las familias con estrato económico alto como un medio para preservar el prestigio social y económico (UNICEF, 2014).

En México, Frías (2017) documenta, con base en la Encuesta Nacional sobre la Dinámica de las Relaciones en los Hogares (ENDIREH 2011) y la Encuesta Nacional sobre Salud y Derechos de las Mujeres Indígenas (ENSADEMI 2008), que 4.1 por ciento de las mujeres indígenas han sido obligadas a casarse y 4.8 por ciento fueron compradas con fines matrimoniales. También señala que 1.8 por ciento de las mujeres en regiones indígenas fueron casadas sin su consentimiento y 2.3 por ciento fueron raptadas para posteriormente casarse con ellas.

Los estudios etnográficos en México realizados en las poblaciones indígenas describen que el matrimonio arreglado consiste en que los padres comprometen en matrimonio a sus hijos e hijas "entre las edades de 14 y 18 en las mujeres y entre los 16 y 20 en los hombres, lo que permite una vida normal sin represiones y conflictos" (Pozas, 2012:113). Una de las características del matrimonio arreglado indígena es que las niñas y adolescentes tradicionalmente, deben ser "pedidas y entregadas en matrimonio", si bien puede ser con su consentimiento o no, mientras que los hombres tienen la oportunidad de elegir con quien se van a casar y que sea de su agrado, lo 
cual significa una "asimetría entre la condición de la mujer y del hombre ante el matrimonio" (Bourdieu, 2007: 312). Aunque las mujeres tuvieran la oportunidad de rechazar casarse con el pretendiente cuando es pedida porque no es de su agrado, no siempre es aceptado su rechazo, puesto que en la mayoría de las culturas el matrimonio "representa una alianza entre familias, un instrumento de cohesión social y por lo tanto, no existen consentimientos individuales, sino procesos de negociación y de pactos colectivos" (Igareda, 2015: 616).

Por otra parte, Gonzáles Montes y Mojarro Iñiguez (2011) en su estudio de ocho regiones indígenas de México, reportan que la región Altos de Chiapas es la que tiene mayor porcentaje de novias pedidas 72 por ciento, robadas 18.8 por ciento y compradas 8.6 por ciento, mientras que en la región maya ${ }^{2}$ únicamente 1.1 por ciento fueron compradas (Gonzáles y Mojarro, 2011). Sin embargo, aunque hay un porcentaje alto de novias pedidas o compradas, muchas de ellas pudieron haber casado sin su consentimiento, es decir, que fueron obligadas a hacerlo. ${ }^{3}$ Gonzales, 1999 (citado en Gonzáles y Mojarro, 2011:193) argumenta que con relación a la compra-pago de la novia "se refiere a que el novio y la familia deben entregar dinero y/o diversos bienes a los padres de la novia, como compensación por los gastos en los que incurrieron en la crianza de la muchacha, dado que ella se trasladará a vivir al hogar de sus suegros".

Ante la alarmante cifra de más de seis millones de mujeres entre 12 y 17 años que en México han contraído matrimonio o viven en concubinato, apenas el pasado 30 de abril de 2019, la Cámara de Diputados en México, derogó 29 artículos del Código Civil Federal para suprimir todas las menciones al matrimonio entre menores de edad (Cámara de Diputados, 2019). El estado de Chiapas, ocupa el primer lugar nacional en matrimonio infantil debido a que 44.82 por ciento de las mujeres han contraído matrimonio antes de cumplir 18 años de edad, seguido de Guerrero, 42.41 por ciento y Oaxaca, 39.17 por ciento (El Universal, 2019).

En cuanto al embarazo adolescente, ha sido un tema ampliamente estudiado en América Latina. Se ha demostrado que la maternidad y los embarazos adolescentes ocurren con mayor frecuencia en los estratos sociales bajos, sectores marginados y pobres, en los que se encuentra sobrerrepresentada la población indígena (Menkes y Suárez, 2003; Stern, 2004; Arias et al., 2008). El embarazo adolescente está "estrechamente relacionado

${ }^{2}$ Región Maya se refiere a cuatro municipios del estado de Yucatán: Chemax, Mérida, Oxkutzcab y Valladolid (Valdez-Santiago et al., 2008).

${ }^{3}$ Torres (2015: 838) señala que "no todos los matrimonios arreglados son forzados, todos los matrimonios forzados son, a su vez, concertados". 
con las normas culturales que prevalecen entre las indígenas, donde la unión tiene que estar inmediatamente asociada al embarazo" (Chávez et al., 2007: 46; Mojarro et al., 2008). En el caso de las indígenas, se ha señalado que los padres entregan en matrimonio a sus hijas cuando son muy jóvenes, mediante lo que se conoce como matrimonio arreglado, concertado o pactado (Mindek, 2003; Araiza, 2006; Torres, 2015); aunque también existe el matrimonio o unión "voluntaria" de los y las menores debido a que los patrones culturales han legitimado los matrimonios y uniones a edades muy jóvenes. Las mujeres indígenas tienen pocas oportunidades de acceso a la educación y ocupación remunerada, por lo que las alternativas a la maternidad son escasas (Menkes y Stern, 2012). De ahí que se considere que el embarazo a temprana edad es un objetivo buscado, deseado, que ayuda a concretar su único proyecto de vida: el de ser madres y, en la mayoría de los casos, esposas (De Jesús, 2011; Menkes y Suárez, 2013; Solís, 2013).

El embarazo es un objetivo individual, familiar y social sujeto a normas de género que pueden sancionar a la mujer que no se embaraza enseguida de la unión: "si la mujer no se embaraza en un plazo acordado" (Araiza, 2006:5) "el marido puede regresarla con sus padres o en su defecto llevar a vivir con su familia a una segunda esposa" (Freyermuth et al., 2014:19). Ojeda (1999:38), señala que "es común que en nombre de la preservación de los intereses de la unidad familiar y comunitaria se justifique socialmente reglamentaciones y prácticas jurídicas que, en muchas ocasiones, funcionen en detrimento de los intereses de los individuos", y que estos intereses son más fuertes en contextos indígenas donde existen prácticas culturales muy arraigadas y que operan a través de las normas sociales. Entre estos intereses, la vida sexual y reproductiva de las mujeres es más afectada y vulnerada, porque "la mujer solo puede tener relaciones eróticas coitales en el matrimonio y solo puede ser madre en el matrimonio" (Lagarde, 2011:335). Con frecuencia las mujeres indígenas se ven sometidas a prácticas sexuales y reproductivas no deseadas, porque las normas de género dictan que ser mujer significa ser esposa y madre; "una mujer debe ser esposa siempre para asegurar su maternidad culturalmente aceptada" (Lagarde, 2011: 335).

El Fondo de Población de las Naciones Unidas (UNFPA), reconoce realidades como la señalada en párrafos anteriores, y documenta que los embarazos en adolescentes tienden a ocurrir con más frecuencia en minorías étnicas, y que son el resultado de un conjunto de elementos que interactúan, como la pobreza y la aceptación del matrimonio infantil por parte 
de las comunidades y la familia (UNFPA, 2013). Ese organismo afirma que las mujeres menores de 18 años aún no tienen la capacidad de tomar decisiones sobre su vida reproductiva, así como decidir sobre el número de hijos y con qué frecuencia, y reconoce que la gestación adolescente es el resultado de la falta de poder de decisión y de la presión social de su entorno, además de la falta de otras opciones de vida.

Este estudio, realizado en la comunidad indígena Tseltal Amatenango del Valle en Chiapas, describe la condición, y cambios del matrimonio forzado y del embarazo adolescente en un contexto de rígidas normas de género, pero en el que se han registrado cambios económicos y educativos para las mujeres. En la primera parte del documento se describen algunas de las características sociodemográficas y económicas de la comunidad, su situación actual y sus transformaciones en las últimas décadas. Con ello se pretende contextualizar el descenso de la práctica del matrimonio forzado, que se ha acompañado de otros cambios reproductivos como la disminución de la edad de unión y retraso en el primer embarazo en adolescentes unidas. Finalmente, se discute el cambio en estas prácticas a la luz de algunos supuestos sobre la disminución del matrimonio forzado como resultado del reconocimiento de los derechos de las mujeres indígenas y de una mayor equidad de género, así como el posible impacto que tendría la modificación de la legislación para prohibir el matrimonio infantil en México.

\section{Aspectos Metodológicos}

Para realizar este estudio se recurrió a metodologías cuantitativas y cualitativas, así como a la revisión de fuentes secundarias de información. Este estudio contó con la aprobación del Comité de Ética en la Investigación de El Colegio de la Frontera Sur. En el apartado cuantitativo, el acopio de información fue realizado mediante una encuesta probabilística aplicada a 120 hogares en cuatro Áreas Geoestadística Básica (AGEB) en la cabecera municipal de Amatenango del Valle. Fueron diseñados dos cuestionarios específicamente para este estudio: el primero, aplicado a familias que contiene preguntas para obtener la estructura familiar y datos sociodemográficos de sus integrantes, así como condición de salud, trabajo y aporte al gasto familiar; el segundo, correspondió a un cuestionario individual dirigido específicamente a mujeres de 12 a 55 años de edad alguna vez unidas y alguna vez embarazadas, en el que fueron preguntas para reconstruir la historia de embarazos, las historias de la unión y del primer embarazo, la condición actual de unión, ocupación, ingresos, y percepción del cambio o no, de la condición de las mujeres de mayor edad, respecto a las más 
jóvenes. Ambos cuestionarios fueron sometidos a pruebas piloto en una comunidad diferente a la de estudio, pero cercana y semejante en cuanto a la etnia, para adecuar el lenguaje, la extensión y la comprensibilidad de las preguntas por parte de las mujeres entrevistadas, a la vez que lograr la capacitación de los encuestadores y encuestadoras. La información recopilada fue capturada en dos bases de datos, correspondientes a cada uno de los cuestionarios.

El matrimonio forzado fue identificado mediante la pregunta ¿usted quería casarse? (cuando se casó), que corresponde a la definición de matrimonio forzado expresada por la UNICEF (2014), descrita en páginas previas. ${ }^{4}$ Seguido de una serie de preguntas sobre las circunstancias en que ocurrió el matrimonio, incluyendo si fue "pedida", si conocía al novio antes de que fuera pedida, si se fugó con el novio sin consentimiento de los padres, entre otras. El embarazo adolescente, fue estimado a partir de los datos obtenidos por la historia de embarazos.

El análisis de los datos fue realizado para describir la magnitud y tendencias del matrimonio forzado, así como de la edad de unión, características de las uniones, la escolaridad y el trabajo remunerado. Adicionalmente, mediante análisis de regresión logística binaria se estableció un modelo explicativo del embarazo adolescente, en el que se incluyeron como variables independientes la escolaridad, el trabajo remunerado y el deseo o no de contraer matrimonio (si quería casarse o no). Esta última variable fue utilizada para describir y comparar el cambio reproductivo, tomando en cuenta las consideraciones conceptuales sobre matrimonio forzado y matrimonio "voluntario" descritas previamente.

El apartado cualitativo consistió en dos grupos focales para comprender las condiciones y razones de las mujeres para aceptar o no el matrimonio cuando "son pedidas", y lo que piensan sobre el matrimonio forzado; todo ello en relación con sus actividades económicas remuneradas. En el primer grupo focal participaron ocho mujeres y, en el segundo, cinco mujeres. Ambos fueron realizados en español ya que gran mayoría de las mujeres en la comunidad son bilingües y la totalidad de quienes participaron en los grupos focales hablan y comprenden bien el español. La información fue grabada, previo consentimiento de las participantes y, posteriormente fue transcrito para su análisis, garantizando la confidencialidad de los datos y de las participantes.

\footnotetext{
${ }^{4}$ El matrimonio forzado es "todo aquel que se celebra sin el consentimiento pleno y libre de al menos uno de los contrayentes, debido a coacciones o a una intensa presión social o familiar" (UNICEF, 2014: 4).
} 
Matrimonio forzado y embarazo adolescente en indígenas en Amatenango del Valle, Chiapas ... / J. LUNA PÉREZ et al.

\section{EL CONTEXTO DE ESTUdio}

Amatenango del Valle, que es uno de los 17 municipios de la región Altos Tsotsil-Tseltal de Chiapas. Está ubicada sobre la carretera Panamericana a 32 kilómetros al sur de San Cristóbal de las Casas (Figura 1) que es la ciudad más importante de la región Altos.

La cabecera municipal de Amatenango del Valle ocupa un lugar estratégico, ya que se puede comunicar directamente con dos ciudades principales: San Cristóbal de las Casas y Comitán de Domínguez mediante la carretera internacional, lo que ha permitido el intercambio comercial de ciertos productos y artesanías.

Para 2015, Amatenango del Valle tenía una población total de 5,071 habitantes, de los cuales 46.6 por ciento son hombres y 53.4 por ciento son mujeres. Hay un total de 1,010 adolescentes de 10 a 19 años de edad, 48.5 por ciento son hombres y 51.8 por ciento son mujeres (INEGI, 2015). Es un municipio de alto grado de marginación, 67.57 por ciento de la población vive en pobreza extrema (Coneval, 2010). El idioma predominante es el tseltal, 97 por ciento de sus habitantes se considera indígena, de ellos 8.5 por ciento son monolingües de tseltal, es decir, no hablan español (INEGI, 2015).

Respecto a la cobertura de servicios de salud, en la cabecera municipal de Amatenango del Valle se cuenta con una unidad médica rural de consulta externa que pertenece al Instituto Mexicano de Seguro Social (IMSS-Prospera), que comenzó a operar a finales de la década de 1970 y que brinda, de manera gratuita, consultas de medicina general, atención materno-infantil, planificación familiar, educación para la salud, promoción del saneamiento y orientación nutricional, además de inmunizaciones, control de enfermedades transmisibles, detección oportuna del cáncer, enfermedades crónico-degenerativas, farmacia, archivo y estimulación temprana.

Una de las acciones más importante que se realiza en esta unidad es la de promover el uso de métodos anticonceptivos a todas las mujeres que acudan a solicitar atención médica; actualmente, un porcentaje importante (46.9 por ciento) de las mujeres en edad reproductiva llevan su control de planificación familiar en esta unidad. Cabe mencionar que en esta unidad se encuentra un Centro de Atención Rural al Adolescente (CARA), que inició sus actividades en 1998. Este Centro inicialmente funcionaba de manera regular impartiendo pláticas en las escuelas sobre prevención de embarazos adolescentes. 
Figura 1: Localidades e infraestructura del municipio de Amatenango del Valle, Chiapas

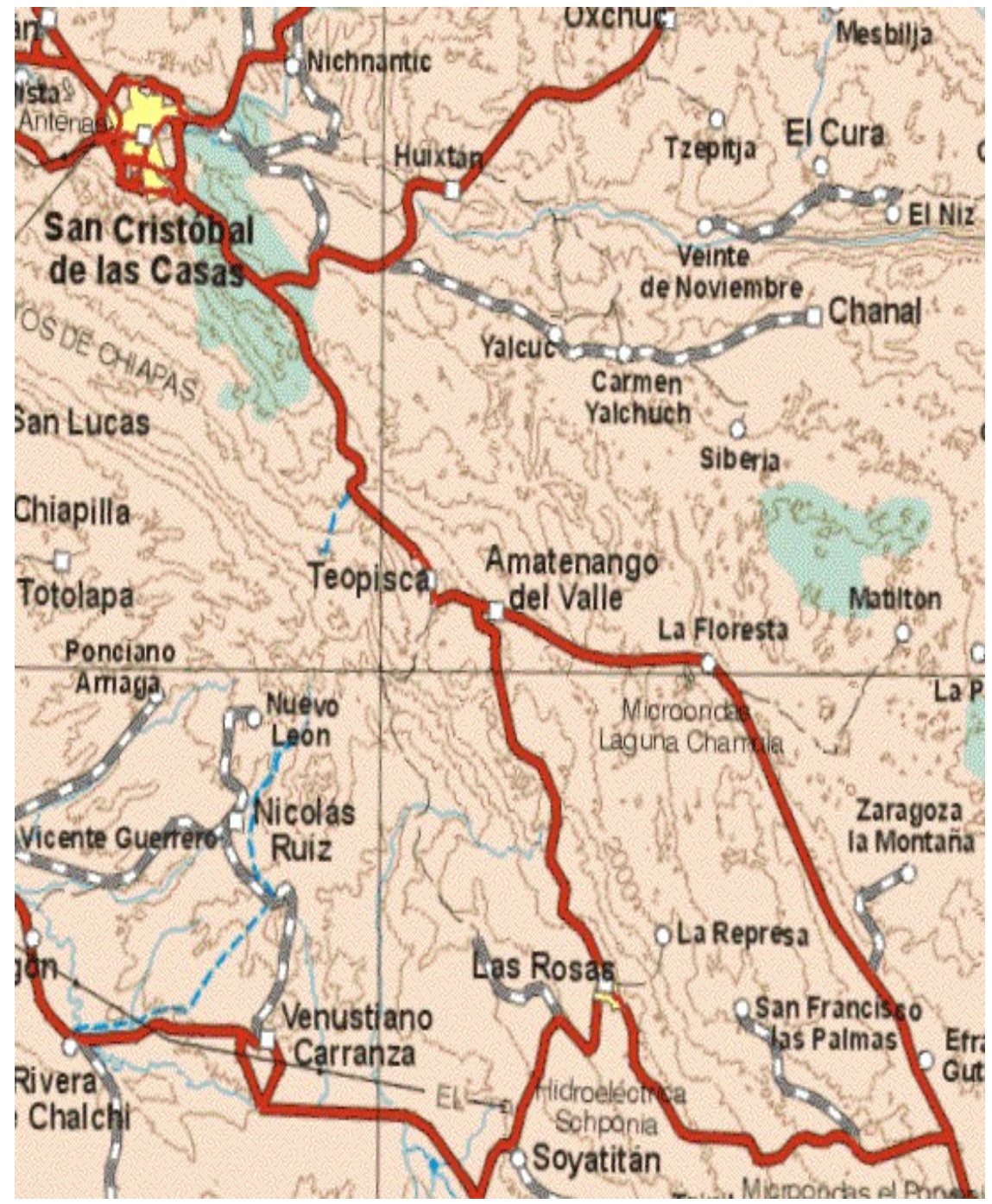

Fuente: Microsoft Bing. mapa de Amatenango del Valle Chiapas - Bing. Actualizado el 04-05-21 
Actualmente, este espacio no está en funcionamiento debido a la falta del personal para realizar actividades y proporcionar atención a los adolescentes y jóvenes, y también por las presiones de las iglesias locales, por lo que las y los adolescentes que radican en este lugar no reciben información sobre salud sexual reproductiva y prevención de embarazo adolescente, $\mathrm{y}$ menos aún cuentan con información sobre métodos anticonceptivos y la disponibilidad de los mismos. Por ello, las actividades de planificación familiar se dirigen únicamente a mujeres unidas, pero no a mujeres y hombres solteros, aunque tengan una vida sexual activa. La unidad médica rural cuenta con un médico pasante y una técnica de enfermería con plaza definitiva en turno matutino; los fines de semana el servicio médico es ofrecido por una médica de contrato.

En cuanto a la infraestructura educativa, en la cabecera municipal existen nueve escuelas preescolares y ocho escuelas primarias distribuidas en diferentes barrios. Hay escuelas federales-monolingües, indígenas-bilingües y del sistema $\mathrm{CONAFE}^{5}$ en donde los niños y las niñas tienen acceso a recibir los cursos de educación básica. En 1988 se inauguró una escuela secundaria, pero fue hasta 1997 que se consolidó con una población estudiantil de 30 alumnos (Ramos, 2003). En el ciclo escolar 2001-2002 abrió sus puertas el plantel No. 256 del Colegio de Bachilleres de Chiapas, lo que permitió a las y los jóvenes continuar con sus estudios en el nivel medio superior en su propia comunidad. Antes de la llegada del plantel, los jóvenes que querían continuar con sus estudios lo hacían en Teopisca municipio vecino de Amatenango. Es importante mencionar que en esta comunidad hay jóvenes que han emigrado a otras ciudades del estado o fuera del estado para continuar con sus estudios universitarios, se encontraron testimonios de padres de familia que tienen hijos e hijas estudiando en la ciudad de México, Morelia, Veracruz, y dentro del estado, se encontraron principalmente ciudades de San Cristóbal de las Casas y Comitán, por lo que se registran jóvenes que han concluido sus estudios universitarios. No obstante, sigue habiendo un porcentaje importante de población de 6 a 14 años que no asiste a la escuela (nueve por ciento); 27.4 por ciento de la población de 15 años o más es analfabeta, y 28.9 por ciento de las personas de 15 años o más, tienen la educación básica incompleta. Durante el ciclo escolar 2014-2015, en el nivel primaria había 1,655 alumnos inscritos en la

\footnotetext{
${ }^{5}$ El Consejo Nacional de Fomento Educativo se creó el 11 de septiembre de 1971 como un organismo público descentralizado de la Secretaría de Educación Pública (SEP) de México, con personalidad jurídica y patrimonio propios. El principal objetivo es generar equidad educativa, para los niños y jóvenes de sectores vulnerables del país. Combatir el rezago educativo en educación inicial y básica. Fecha de consulta, 7/05/2019. Disponible en https://es.wikipedia.org/wiki/ Consejo_Nacional_de_Fomento_Educativo
} 
escuela, de los cuales 50 por ciento eran hombres y 50 por ciento mujeres; en la secundaria, en total había 496 estudiantes, de ellos 59 por ciento eran hombres y 41 por ciento eran mujeres; en el nivel bachillerato había 206 alumnos, 72 por ciento eran hombres y 28 por ciento mujeres (Anuario Estadístico y Geográfico de Chiapas, 2016), documentándose que, conforme avanza el nivel de escolaridad, el porcentaje de mujeres es menor. Como se analizará enseguida, esta desigualdad se encuentra relacionada con la dinámica económica de la comunidad, en la que las mujeres ocupan un lugar destacado.

Entre las principales actividades económicas y fuentes de ingreso destacan la alfarería artesanal y textil, migración temporal de hombres a ciudades cercanos o a otros estados del país y fuera del país; la venta de aves, ganado y hortalizas de traspatio, el empleo temporal en las oficinas del municipio, así como la producción de maíz y frijol. Se practica en menor medida la ganadería de traspatio, y parte de sus ingresos se complementa con los apoyos gubernamentales como Procampo ${ }^{6}$ y Prospera. ${ }^{7}$ Existe una clara división sexual del trabajo, los hombres se dedican en las actividades agrícolas, mientras que las mujeres se dedican principalmente a las actividades artesanales de alfarería y textil, combinándolas con las actividades domésticas y el cuidado de sus hijos. Hay dos colonias en donde las mujeres únicamente se dedican a elaborar ropa artesanal que a van a vender a San Cristóbal de las Casas y Comitán, actividades que les genera escasos ingresos económicos, por lo que ellas también participan en las actividades agrícolas y el cuidado de ganado de traspatio.

De acuerdo con Nash (1993) y Ramos (2003), el trabajo de alfarería se ha ido transformando con el tiempo, aunque desde la época prehispánica se

\footnotetext{
${ }^{6}$ Programa de Apoyos Directos al Campo tiene como objetivo es complementar el ingreso económico de los productores del campo mexicano, ya sean de autoconsumo o de abastecimiento, para contribuir a su crecimiento económico individual y al del país en su conjunto; así como incentivar la producción de cultivos lícitos, mediante el otorgamiento de apoyos monetarios por superficie inscrita al Programa, de acuerdo a lo que establece la normatividad vigente; coadyuvando así a la atención de las necesidades respecto al derecho a la alimentación, planteadas en el Pacto por México, fecha de consulta, 26/02/2019. Disponible en http://www.sagarpa.mx/ agricultura/Programas/procampo/Paginas/Objetivo.aspx

7 El 5 de septiembre de 2014, a través de un Decreto Presidencial, Oportunidades se fortalece y se transforma en PROSPERA Programa de Inclusión Social, cuyo objetivo es articular y coordinar la oferta institucional de programas y acciones de política social, incluyendo aquellas relacionadas con el fomento productivo, la generación de ingresos, el bienestar económico, la inclusión financiera y laboral, educación, alimentación y salud, dirigidas a la población en situación de pobreza, bajo esquemas de corresponsabilidad que les permitan a las familias mejorar sus condiciones de vida y asegurar el disfrute de sus derechos sociales y el acceso al desarrollo social con igualdad de oportunidades, fecha de consulta, 26/02/2019. Disponible en https://www.gob. $\mathrm{mx} /$ prospera/documentos/que-es-prospera

A partir del cambio sexenal en el gobierno federal (2018-2024), ninguno de los dos programas siguió operando.
} 
practicaba para elaborar utensilios de uso doméstico o para ceremonias religiosas, fue "a partir de finales de 1980 que la comercialización de artesanía adquiere prioridad en la comunidad y que la llegada masiva de turistas al estado abrió también otra forma de comercializar" (Ramos, 2003: 19). Además de la reciente apertura de la carretera panamericana, abrió las posibilidades de instalar comercios de alfarería en las orillas de la carretera, lo que también facilitó el acceso a turistas y la movilización de las mujeres alfareras para ofrecer sus productos a otras ciudades de Chiapas.

A finales de la década de 1970 y a principios de la siguiente se inició un profundo cambio económico en la comunidad. En ese periodo había una constante emigración masculina por la crisis agrícola y económica, lo que originó la reestructuración de las actividades productivas de las familias y principalmente de las mujeres. Las dificultades económicas que vivían las familias obligaron a los hombres a emigrar a ciudades como Villahermosa, Tuxtla Gutiérrez y San Cristóbal de Las Casas, para trabajar de peones o albañiles (Collier, 1992). Otros iban a trabajar en la zafra de caña de azúcar a Pujiltic, mientras que las mujeres, para generar ingresos salían a vender sus productos artesanales de alfarería en ciudades cercanas como Tuxtla Gutiérrez, Comitán y San Cristóbal, y los principales productos eran macetas, comales y cántaros destinados a uso utilitario o decorativo (Ramos, 2003).

Sin embargo, Nash señala que el poder salir a otros lugares, vender sus productos y obtener ingresos económicos directamente, afectaba la posición de las mujeres, lo que provocó "usar medios más poderosos de supresión para preservar las relaciones patriarcales" (Nash, 1993: 148). Cabe mencionar que no solo por eso fue el descontento de los hombres sino también por el trabajo organizado de las mujeres en una cooperativa fomentada por el Instituto Nacional Indigenista, porque implicaba trabajar en los talleres y en grupos, idea que no fue agradable para los hombres hasta que la cooperativa desapareció por la "resistencia masculina"; sin embargo, la participación económica de las mujeres en la alfarería no disminuyó, e incluso se ha incrementado con el tiempo.

A pesar de las dificultades y diferentes obstáculos que enfrentaban las mujeres de Amatenango, de acuerdo con Ramos (2003: 18): “ellas iban ganando un estatus diferente dentro del contexto indígena de los Altos. Ellas, siendo indígenas, viajaban a la ciudad, aprendieron a hablar español y tenían experiencia en los espacios públicos", lo que, de acuerdo con los resultados de este estudio, ha sufrido un retroceso importante en cuanto a salir de la comunidad, en la medida en que la producción de la alfarería y 
su venta local, ha sido cada vez más importante, manteniéndose ahora con mayor frecuencia en su comunidad de origen.

Amatenango del Valle es, con base en datos del Censo de Población y Vivienda, 2010, el municipio indígena de los Altos de Chiapas con la más alta tasa de participación económica de las mujeres de 12 años y más: 37.17 por ciento. ${ }^{8}$ En los últimos años las mujeres alfareras, en producción individual y familiar, se han visto en la necesidad de innovar sus productos para poder competir en el mercado (Ramos, 2003). En este estudio se observó competencia entre mujeres y entre familiares en la producción de jaguares, pavorreales, palomas, gallos y otros productos más modernos, destinados específicamente para el mercado turístico.

El esfuerzo creativo busca que no se repitan los diseños de sus vecinas o de otras mujeres, y son las hijas, principalmente, quienes participan fuertemente en la creación de nuevos productos, desde la fabricación, la decoración y el diseño de los colores. Las niñas entre los 8 y 12 años empiezan hacer las piezas de barro, comienzan con figuras muy pequeñas hasta que van aprendiendo hacer objetos más grandes. A partir de los 15 años elaboran sus propias piezas y las comercializan. Los ingresos obtenidos de la venta de la alfarería se destinan para la compra de alimentos y ropa para la familia, así como para herbicidas y fertilizantes; además de la contribución a la economía familiar, tiene un fuerte impacto como recurso de resistencia ${ }^{9}$ frente a las presiones de género ejercidas por los varones en la comunidad, en particular en lo relativo a contraer matrimonio.

\footnotetext{
8 Esta cifra es menor a los resultados obtenidos en el trabajo de campo, más de 80 por ciento de las mujeres tienen trabajo remunerado. Sin embargo, el INEGI no obtiene esta información dado que la mayoría de las mujeres valoran poco los trabajos artesanales que realizan y suelen decir que trabajan en el hogar, espacio donde ellas elaboran sus productos artesanales para vender. De acuerdo con INEGI (2010), las mujeres de 12 años o más en Amatenango del Valle registran la mayor tasa de participación económica (37.17 por ciento) después de San Cristóbal de las Casas (en su mayoría población mestiza): (38.7 por ciento). En los demás municipios de la región Altos Tsotsil-Tseltal la participación económica de las mujeres es: Chamula, 28.50 por ciento, Chenalhó 22.22 por ciento, Huixtán 21.99 por ciento, Chanal 18.55 por ciento, Larráinzar, 16.23 por ciento, Teopisca, 16.15 por ciento, Tenejapa 16.23 por ciento, Chalchihuitán, 10.92 por ciento, Zinacantán, 10.03 por ciento, San Juan Cancuc, 9.72 por ciento, Oxchuc, 9.04 por ciento, Aldama, 6.98 por ciento, Pantelhó, 6.63 por ciento, Mitontic, 5.19 por ciento, Santiago el Pinar, 3.43 por ciento (Censo de Población y Vivienda, 2010, INEGI).

9 La posibilidad de negociación reproductiva en mujeres rurales a partir de la obtención de recursos propios ha sido tratada por Bina Agarwal (1999) y, en el caso de Chiapas, para población rural no indígena, por Ramírez y colaboradores (2005).
} 
Matrimonio forzado y embarazo adolescente en indígenas en Amatenango del Valle, Chiapas ... / J. LUNA PÉREZ et al.

\section{Resultados}

\section{Tendencias socioeconómicas y cambio reproductivo}

En relación con la actividad económica, se observó que la mayoría de las mujeres ( 81.7 por ciento) ha realizado actividades remuneradas alguna vez, con porcentajes elevados en todos los grupos de edad, sin diferencias significativas entre ellos $(p=0.084)$ (Figura 2).

Adicionalmente, se observa que las mujeres adolescentes unidas registran en mayor proporción que las no unidas el desempeño de un trabajo remunerado (88.5 vs. 66.7 por ciento), respectivamente $(\mathrm{p}=0.032)$; mientras que en los otros grupos de edad no se registraron diferencias estadísticamente significativas. El unirse en esta comunidad significa asumir todas las responsabilidades asociadas a ser esposas y madres y cumplir con las normas sociales que la comunidad establece, incluyendo el trabajo remunerado para contribuir a la economía familiar, lo que se considera parte de sus obligaciones. ${ }^{10}$

Respecto a la escolaridad, se aprecia un cambio significativo por grupos de edad, en el que las mujeres más jóvenes (15 a 19 años) tienen en promedio mayor escolaridad que las mujeres de mayor edad $\left(X^{2}=197.634\right.$, $12 \mathrm{gl}, \mathrm{p}<0.000$ ); asimismo, se observa una reducción en la proporción del matrimonio forzado $\left(\mathrm{X}^{2}=16.321,6 \mathrm{gl}, \mathrm{p}=0.012\right)$, y una disminución en la edad de unión $\left(\mathrm{X}^{2}=41.445,18 \mathrm{gl}, \mathrm{p}=0.001\right)$ (Figura 3).

En la Tabla 1, se muestran las cifras de la edad media de unión de las mujeres, la edad media de unión de los esposos, la proporción que se unieron antes de cumplir 18 años, el porcentaje de matrimonio forzado (no querían casarse) y el porcentaje de unión antes de cumplir 18 años entre quienes no querían casarse, por grupos de edad.

En total, de las 340 mujeres unidas entrevistadas de 15 a 49 años, 4.7 por ciento correspondió a matrimonios forzados; de ellas, 25.0 por ciento ocurrieron cuando las mujeres eran menores de 15 años (12-14 años), 66.7 por ciento cuando tenían entre 15 y 19 años y 8.3 por ciento cuando ellas tenían entre 20 y 24 años. Estos datos indican que no todos los matrimonios forzados corresponden a matrimonio infantil o adolescente.

\footnotetext{
${ }^{10}$ La dinámica económica en la que las mujeres tienen una importante participación, en un contexto de desigualdad y violencia de género, son condiciones que, han generado acciones de resistencia de las mujeres a la violencia ejercida por los varones, mediante la renuncia de un porcentaje importante de mujeres a la nupcialidad y, por consecuencia a la maternidad, porque la conyugalidad involucra la maternidad (Linares, 2018).
} 


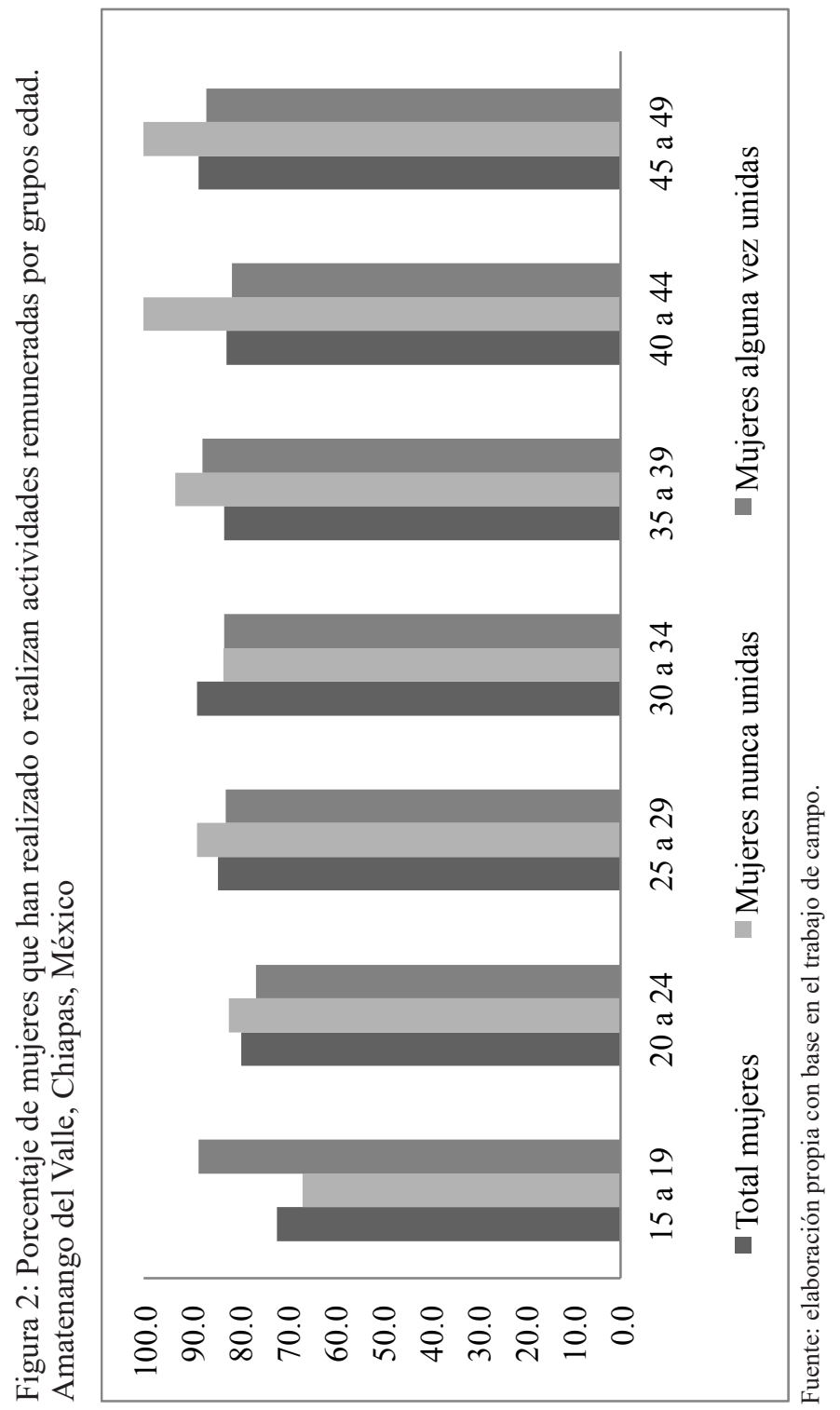


Matrimonio forzado y embarazo adolescente en indígenas en Amatenango del Valle, Chiapas ... / J. LUNA PÉREZ et al.

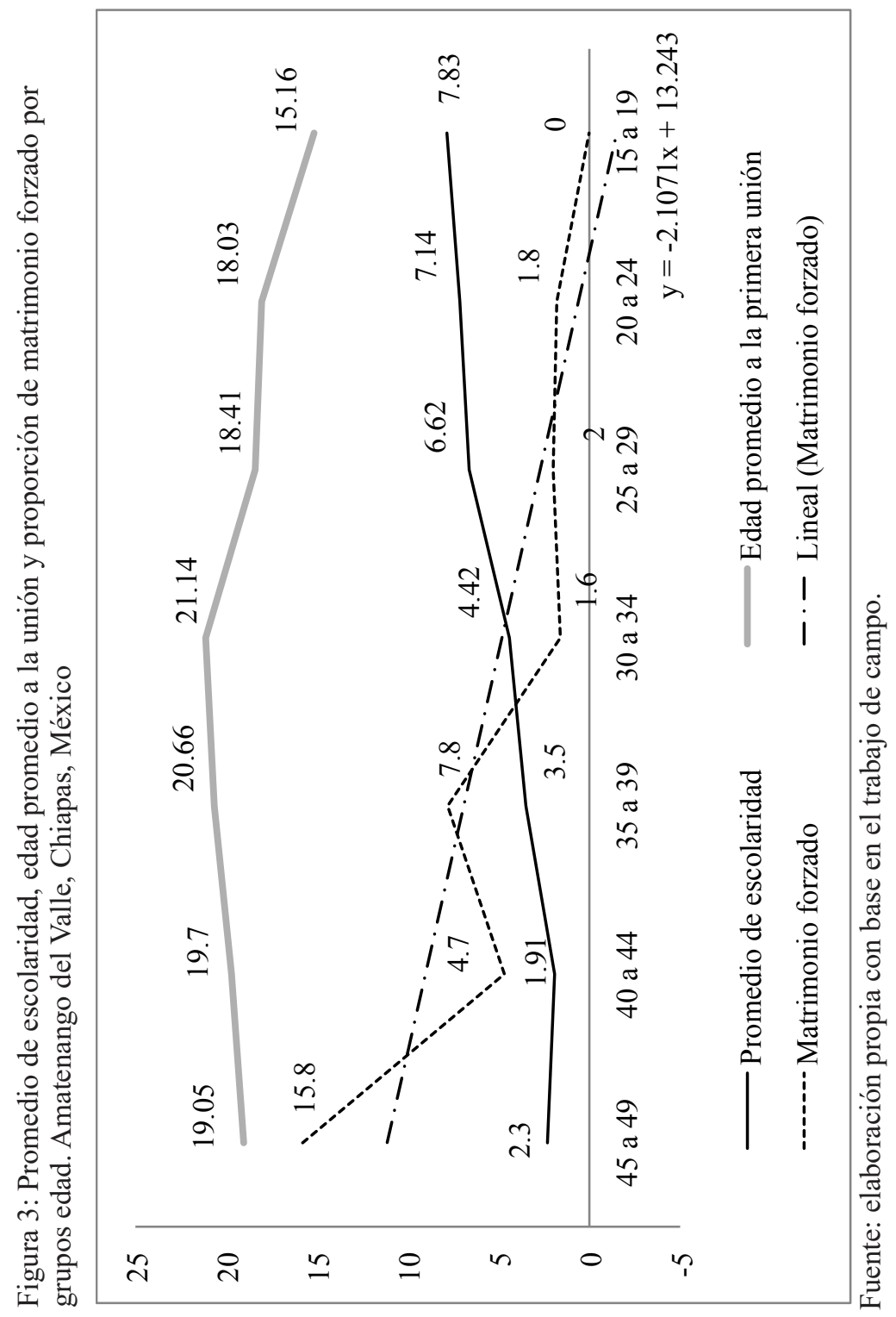




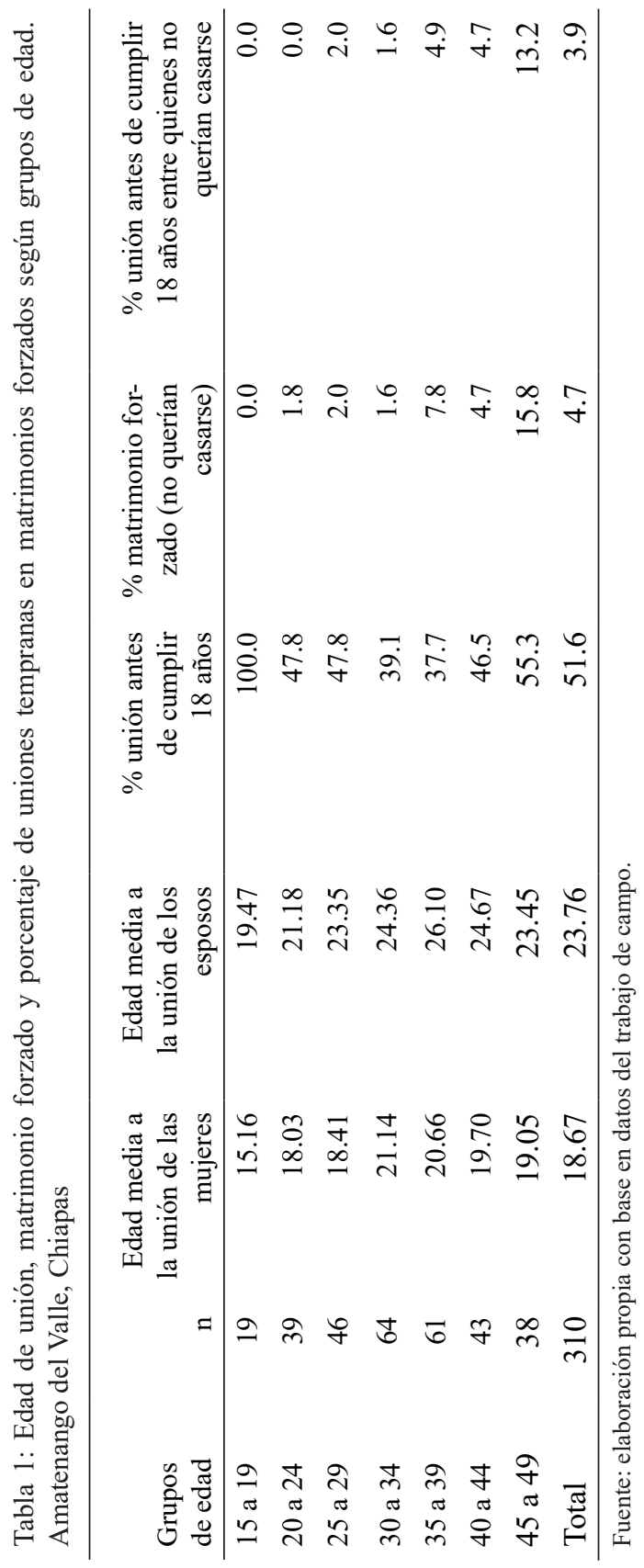


Sin embargo, el mayor porcentaje se observa cuando las adolescentes se encontraban en el rango de edad de 15 a 19; el matrimonio forzado infantil ocurrió en uno de cada cuatro matrimonios forzados.

También se observa que existe una relación entre el matrimonio forzado con el embarazo adolescente, registrándose que entre las mujeres que no querían casarse, 59.1 por ciento tuvo su primer hijo/a antes de cumplir 18 años, cifra significativamente mayor a la de las mujeres que se sí querían casarse: 30.7 por ciento $\left(X^{2}=10.127,4 \mathrm{gl}, \mathrm{p}=0.038\right)$. Lo anterior ocurre porque la edad de unión se encuentra estrechamente relacionada con la edad a la que ocurre el primer embarazo $\left(\mathrm{X}^{2}=159.186,3 \mathrm{gl}, \mathrm{p}=0.001\right)$, y como se ha señalado, un elevado porcentaje de los matrimonios forzados ocurrieron cuando las mujeres eran menores de edad.

La relación entre unión y embarazo temprano en las mujeres que fueron forzadas a contraer matrimonio puede explicarse como resultado no solamente por las escasas alternativas a la maternidad de las mujeres indígenas (Menkes y Stern, 2012), quienes, una vez unidas, son presionadas por las normas culturales para embarazarse enseguida (Chávez et al., 2007; Mojarro et al., 2008), sino por la condición de desventaja por su edad que facilita el ejercicio del poder y la imposición de los padres, la pareja y la comunidad para que establezca una unión no deseada sin más opción que sujetarse a las normas sociales establecidas.

El matrimonio forzado en menores de edad ocurrió con un hombre al que no conocían, siempre de mayor edad que ellas, y donde la violencia ejercida por este sobre la esposa es una referencia común en la comunidad. Algunos testimonios de las mujeres dan cuenta de ello:

"Yo me casé cuando tenía 15 años (...) y luego encontré mi bebé".

— ¿Usted decidió casarse a esa edad?

"No, no quería yo pue, no quería, pero mi abuelito, mi abuelito, como me entraron a pedir, ya te entraron a pedir, yo lo voy a recibir el bocado me decía mi abuelito, yo no quería yo pue todavía, pero a la fuerza me dijeron, (...)".

— ¿Usted conoció antes a su esposo?

"No, no conocía yo, ni lo había visto, si, ni lo había visto, pues hasta cuando metieron el bocado [en la pedida se lleva una ofrenda: pan, refresco, cervezas] empezó a llegar en mi casa el muchacho y así lo conocí, iday no quería yo con él, me daba pena, no sé qué me daba, miedo, sí" (Mujer de 55 años. Grupo focal 1, 21 de junio 2018).

-Y usted ¿cómo fue que se casó? 
"Pedida, tenía yo 16 años cuando me entró a pedir [su exesposo], ya mi papá estaba consciente porque ya tenía yo 16 años. Está bien dice, está bien se van a casar, si tú ya pensaste bien, bueno dice el muchacho. Ya me dio ya mi papá”.

— ¿A usted le preguntaron si quería casarse o no?

“Ah no, mi papá no. Nomás dijo pue, salió el hombre, y vino mi papá, mira hija dice te vas a casar con este hombre, ya le dije que si te vas a casar dice, por qué le dijiste si yo no me gusta le dije, no me gusta ese hombre, ni me imaginaba yo que me iba casar con ese hombre, no me gustaba, no es mi destino, no es mi suerte, no me gusta le dije, a poco tú vas a escoger con quien te vas a casar me dijo mi papá, quién sabe soy yo dice, el que manda soy yo me dijo mi papá. ¡Ay! y mi mamá dijo, cómo pudiste hacer esto, si tu hija no quiere es que no quiere, no tú, no, pero va estar bien cerquita su casa el muchacho dijo mi papá, está bien cerca por eso le dije el muchacho que si se van a casar dice, según qué porque vivía cerca, y no, es muy malo su suerte que eligió, pues si porque él lo quiso, yo no, así. Vino el bocado y lo recibieron, y ya después nos casamos un mes de diciembre. Después de dos meses que me casé ya iba balacear el hombre, hasta que llegamos a separarnos" (Mujer de 52 años. Grupo focal 2, 6 de julio 2018.

— ¿Cómo se sintió usted cuando la obligaron a casar?

"Pues para mí — yo dije — ni modo, así es la vida dije yo. Estaba viendo que así era la costumbre pue, antes era normal para las mujeres casarse con un hombre sin haberlo conocido y que los papás decidan por ellas, pero ahora ya sé que ya no es normal" (Mujer de 48 años. Entrevista personal, 5 de mayo 2018).

El descenso en la proporción de matrimonios forzados ha sido significativo en la comunidad, y es reconocido por las mujeres de diferentes edades, quienes expresaron que las muchachas ya pueden elegir y decidir con quién casarse:

"[...] Yo veo que ahora las muchachas ya tienen libertad, ellas deciden si quieren casar o no, si les gusta o no les gusta el muchacho. Ahora ya es libre, ella decide si sí o no, en cambio antes pue aunque no les gustó el muchacho, tienen que aceptar pue, no tiene nada que hacer porque la obligan, la obligan. Así, como que no está bien así" (Mujer de 48 años. Entrevista personal, 5 de mayo 2018).

"[...] ahora ya no es así, ahora ya es más bonito parece"

"[...] y ahora ya ellos se platican, hasta piden permiso [con los papás] algunos muchachos o cuando salen a estudiar ya van conociendo a su novio" (Mujer de 52 años. Grupo focal 1, 21 de junio 2018).

En el mismo sentido fue el siguiente testimonio: 
-Usted ¿cómo fue que se casó?

"Yo [risa]. Pues yo me huí, me fui sola".

-Aaah, te huiste, ¿ya conocías el muchacho, fueron novios?

"Sí".

—Cómo fue que se conocieron?

“Jaja, [todas se ríen]. Lo conocí en el campo, él llegaba a trabajar en su milpa allá por el camino, cuando salía de la secundaría ahí lo veía, él me habló y quise yo también, nos hicimos novios".

— ¿Cuántos meses fueron novios?

"Tres meses, ya estaba terminando la secundaría, ya cuando salí me fui con él". — ¿Cuántos años tenías cuando te fuiste con tu esposo?

“15 años y él tenía 17 años” (Mujer de 22 años. Grupo focal 2, 6 de julio 2018).

Otra mujer que ahora tiene 32 años narró cómo conoció a su esposo y planearon irse a vivir juntos:

"lo que me gustó [de su esposo], jajaja...no es que cuando nos conocimos era muy tranquilo (...), él era músico, tocaba pue, y llegaba a tocar allá en mi comunidad y después él me habló, y yo no le hacía caso, jeje, no le hacía caso porque como no lo veía pue cada ratito, que tal tenía novia o ya tenía esposa, no le hacía caso pero él si no me dejaba ya, jajaja, si pue, después me enamore de él, lo fui queriendo también, es que el corazón manda pue, si una persona quieres no te da miedo, después lo fui conociendo, y así hasta creo que tres años estábamos platicando que me quería, me decía cosas pue y yo también, pero le decía pue mi esposo, le decía, no mira, era mi sueño pues casarme, no salir huyendo, no mira para que no tengamos problemas le voy a decir mi papá mi mamá y que me voy contigo le dije, pero él no quiso, tuvo miedo para decir sí, no, que tal si me regaña tu papá, tu mamá, que tal si no te dejan pue, ah bueno, y me dijo pue si no quieres ir conmigo te voy a dejar, jajaja, y el miedo que tuve, yo lo quería yo también y mejor me vine, jeje.

— ¿Cuántos años tenías cuando te fuiste con tu esposo?

"18 años, mi esposo tenía 21 años" (Mujer de 32 años. Grupo focal 1, 21 de junio 2018.

A partir de estos testimonios se observa que el consentimiento o la unión "voluntaria" se refiere casi en su totalidad a la "elección" de la pareja por ellas y, en un elevado porcentaje, son niñas o adolescentes que establecen la unión sin esperar el consentimiento de los padres. 
En general las mujeres reconocen los cambios en la forma en que establecen la unión las mujeres más jóvenes, aunque también el cambio reproductivo relacionado con la menor edad de unión:

$-[\ldots]$ ¿Y lo siguen haciendo así hasta ahora?

"No, (...). Siempre esta cambiado ya ahora, se huyen pue, ya no lo esperan sus pedidas (...)" (Mujer de 40 años. Grupo focal 2, 6 de julio 2018).

— ¿Hay mujeres que se casan muy chicas?

"Si, tan chiquita, una niña pue que es compañera de mi hijito que va salir de secundaría, ya tiene dos bebés, uno de un año y otro está a punto de nacer o creo que ya nació, ya se alivió creo ya la chamaquita, sí, bien chiquita".

- ¿Ella quiso casarse o la obligaron?

"No, ella quiso, no la obligaron, se huyó pue, ella huyó, se fue. Se fue con un hombre de 26 años, bien grande ya el hombre, pero ni modos ahí se fue la chamaquita. Aquí se casan ya muy chiquitas, a veces no se cuidan, no se cuidan, se van, hay algunas que les gusta casarse así de grande, solo algunas y algunas que se van.

_ ¿A qué edad se fue ella?

"Uuum, creo que, de 14 años, casi llevando dos meses cuando entraron en la secundaría cuando ella se fue, de primer grado de secundaria se fue" (Mujer de 40 años. Grupo focal 2, 6 de julio 2018).

Los cambios en la escolaridad, en la edad de unión, el matrimonio "voluntario" y el retraso del primer embarazo son más evidentes en el grupo de menor edad: las adolescentes. A continuación, se profundiza sobre esos aspectos en las niñas y adolescentes menores de 18 años para comprender las condiciones y características del cambio reproductivo que están experimentando.

\section{El cambio reproductivo en las adolescentes}

En total se obtuvo información por encuesta de 163 mujeres de 12 a 19 años, de las cuales 26 han estado unidas (16 por ciento) y 20 han sido madres (12.3 por ciento); esta última cifra es ligeramente menor a la reportada por el Instituto Nacional de las Mujeres (13.5 por ciento para mujeres en localidades menores a 15 mil habitantes) (INMUJERES, s/f).

Para las adolescentes de menor edad y, en menor medida para las de 17-18 años, la aceptación de la unión por parte de los padres de la novia depende de que se cumplan las prácticas culturalmente establecidas. 
¿Cómo se establecieron estas uniones? 66.7 por ciento fueron pedidas, ${ }^{11}$ 16.7 por ciento fueron robadas con su consentimiento y después pedidas, $\mathrm{y}$ 16.7 por ciento decidieron irse a vivir juntos. De las mujeres adolescentes en la actualidad unidas, solamente dos han formalizado la unión de forma legal. Se encontró que en la totalidad de las que fueron pedidas, los padres estuvieron de acuerdo por la unión, mientras que, en las que primero fueron robadas y luego pedidas, el apoyo de los padres de la novia se registró en un menor porcentaje ( 85.7 por ciento). De las mujeres que declararon haberse ido solas, ninguna tuvo el apoyo de sus padres (Tabla 2) en tanto sanción familiar y social a la transgresión de las normas que exigen que las mujeres sean pedidas. ${ }^{12}$

Tabla 2: Aceptación de la unión por parte de los padres, de acuerdo con la modalidad de unión

\begin{tabular}{|c|c|c|c|c|c|}
\hline \multirow{4}{*}{$\begin{array}{l}\text { ¿Su familia y la de su } \\
\text { esposo estuvieron de } \\
\text { acuerdo en su unión? }\end{array}$} & & orma de unión & & & \multirow{4}{*}{$\begin{array}{l}\mathrm{X}^{2}, \text { gl y } \\
\text { valor } \\
\text { de p. }\end{array}$} \\
\hline & Fue pedida & $\begin{array}{r}\text { Primero fue } \\
\text { robada y } \\
\text { luego pedida }\end{array}$ & $\begin{array}{r}\text { Solo se } \\
\text { fueron juntos }\end{array}$ & Total & \\
\hline & $\mathrm{n}=9$ & $\mathrm{n}=14$ & $\mathrm{n}=3$ & $\mathrm{n}=26$ & \\
\hline & $\%$ & $\%$ & $\%$ & $\%$ & \\
\hline Sí & 100.0 & 85.7 & 0.0 & 80.8 & \multirow{3}{*}{$\begin{array}{l}14.963,2 \\
\text { gl, } 0.001\end{array}$} \\
\hline No & 0.0 & 14.3 & 100.0 & 19.2 & \\
\hline Total & 100.0 & 100.0 & 100.0 & 100.0 & \\
\hline
\end{tabular}

Fuente: elaboración propia con base en datos del trabajo de campo.

El matrimonio formal (civil, por la iglesia o ambos) ocurre con mayor frecuencia entre quienes fueron pedidas (55.5 por ciento), respecto a las que fueron robadas (28.6 por ciento), robadas y después pedidas (19.8 por ciento), o solamente se fueron juntos (10.8 por ciento), a la vez que la separación o abandono ocurrió en cinco por ciento de las mujeres pedidas, en 11.2 por ciento de las que fueron robadas y después pedidas, y en 16.2 por ciento de quienes solamente se fueron juntos. ${ }^{13}$ De esas cifras puede

11 Una de las mujeres pedidas refirió que cuando fue pedida no conocía al pretendiente, lo que significa que se trató de un matrimonio arreglado, pese a que la adolescente reportó que se casó "voluntariamente".

12 El tener relaciones sexuales previas al matrimonio es poco aceptado por los padres y, cuando ocurre, significa un desprestigio social para la familia, por lo que la pareja es rechazada y las mujeres son expuestas a mayor vulnerabilidad y probabilidad de ser abandonadas o separarse.

13 De las mujeres separadas o abandonadas $(n=4)$ todas se unieron entre los 15 y 16 años; sus esposos, en 75 por ciento lo hicieron entre los 20 y 29 años, por lo que se observa una mayor diferencia de edad respecto a la totalidad de uniones, con una diferencia de edad superior de cuatro años; todas tuvieron al menos un hijo, la mitad dijeron que no sabían de los métodos anticonceptivos y el resto no quisieron usarlos. En cuanto a la escolaridad, 75 por ciento concluyeron 
interpretarse lo siguiente: por una parte, el ajustarse a las normas comunitarias y familiares que exigen que las mujeres sean pedidas se asocia con mayor frecuencia al establecimiento de un matrimonio respecto a quienes transgreden esa norma, observándose que quienes deciden vivir juntos, sin pedir a la novia, solamente una de cada diez establece un matrimonio.

Por otra parte, la probabilidad de separación o abandono de mujer por parte de su pareja es significativamente menor entre quienes fueron pedidas respecto a las que no lo fueron. Ambas situaciones hacen evidente el efecto de las normas y el sometimiento institucionalizado, patriarcal, de las mujeres respecto a las formas en que debe establecerse la unión en esta comunidad. Pese a la existencia de apertura comunitaria a las relaciones de noviazgo, entre las jóvenes existe el temor de la negativa de los padres a aceptar como yerno al novio y que se nieguen a darle en matrimonio a su hija, es por eso por lo que existe una práctica muy recurrente "el robo o la huida de la novia"; es decir, las muchachas deciden, al margen de los arreglos familiares, irse con el hombre que ellas han elegido, lo cual es planeado por ambos. Esta forma de establecimiento de la unión es una alternativa al matrimonio arreglado o forzado en el que se manifiesta el temor de que las adolescentes sean entregadas en matrimonio con un hombre al que no conocen o al que ellas no quieren. Si la novia no es pedida, no cuenta con apoyo alguno por parte de los padres lo que las coloca en una posición de mayor vulnerabilidad frente a la pareja y la familia de la pareja, lo que puede resultar en separación y abandono.

La menor edad de las adolescentes es una condición que favorece el que sean sometidas a las decisiones familiares y comunitarias sobre la unión y el embarazo, particularmente en las de 12 a 16 años; $y$, aunque algunas de ellas se atreven a transgredir la norma, son objeto de fuertes sanciones que las colocan en condición de muy alta vulnerabilidad, lo que se agrava en las menores de 15 años.

Según las mujeres, la transgresión de la norma respecto a las formas de unión tiene consecuencias, así como las huidas y unirse muy chicas:

[...] Pero no sirve, a veces solo van a llegar y a veces las regañan, o a veces, saber qué cosas que van a hacer, regresa la muchacha, solo van a ir a tener sus hijitos y se separan, y por qué, porque no esperaron pues sus pedidas, no lo

primaria al igual que sus esposos y 25 por ciento tiene estudios de secundaria al igual que una de ellas. La residencia fue patrilocal. Una de las separaciones correspondió a una pareja que no tuvo el apoyo de los padres, porque decidieron irse juntos sin pedir a la novia. En ese caso el novio tenía 29 años y ella 16. De acuerdo con las experiencias de ellas, opinaron que es mejor que sus hijos e hijas se casen más grandes, después de los 20 años, porque "casarse muy chicas no sirve, luego nos dejan solas". 
saben si es la verdad que quiere mujer el hombre" (Mujer de 40 años. Grupo focal 1, 21 de junio 2018).

"El papá (...) le dijo su hijo que no lo quieren la muchacha, mándalo te voy a buscar otra tu mujer, hay lo mandaron embarazada su sobrina mi esposo, y dice que fueron sus papás que no quisieron la muchacha".

- ¿No aceptaron la muchacha?

"No, no la aceptaron. Si la aceptaron un tiempo, pero después ya no la quisieron".

—PPor qué no la aceptaron?

"Porque su sobrina mi esposo tenía muchos granos en su cara, y dice que con el fuego cuando hacía su tortilla empieza [por el juego le sale más granos] y por eso no quiso sus papás. Si, así regreso la pobre muchacha, si, ya ahorita ya vive con su mamá, ya está grandecita la niña” (Mujer de 48 años. Grupo focal 2, 6 de julio 2018).

No obstante, se aprecian cambios reproductivos y de género en las adolescentes adicionales a la elección de la pareja y de la transgresión de las normas que rigen las uniones, como el posponer el primer embarazo posterior a la unión. De las 26 adolescentes que están casadas o viven en unión libre, seis (23.1 por ciento) dijeron no haberse embarazado porque no querían tener hijos todavía, y reportaron el uso de métodos anticonceptivos hormonales (implantes o inyectables) (Tabla 3).

Tabla 3: Porcentaje de mujeres alguna vez unidas, por grupos de edad, que dijeron "cuidarse" para no embarazarse posterior a la unión (primer embarazo)

\begin{tabular}{lcc}
\hline & $\begin{array}{c}\text { Ocurrencia del primer embarazo } \\
\text { inmediatamente posterior a la } \\
\text { unión }(\text { sí/no) }\end{array}$ & $\begin{array}{c}\text { Antes de embarazarse la } \\
\text { primera vez ise cuidó para no } \\
\text { embarazarse? (sí/no) }\end{array}$ \\
$\begin{array}{l}\text { Mujeres alguna } \\
\text { vez unidas }\end{array}$ & $\begin{array}{c}\% \\
\text { 12 a } 17(\mathrm{n}=26)\end{array}$ & 23.1 \\
18 a $24(\mathrm{n}=55)$ & 78.9 & 21.8 \\
25 a $29(\mathrm{n}=50)$ & 74.0 & 18.0 \\
30 a $34(\mathrm{n}=64)$ & 85.9 & 14.1 \\
35 a $39(\mathrm{n}=64)$ & 79.7 & 18.8 \\
40 a $44(\mathrm{n}=43)$ & 86.0 & 14.0 \\
45 a $49(\mathrm{n}=38)$ & 84.2 & 13.2 \\
\hline
\end{tabular}

Fuente: elaboración propia con base en datos del trabajo de campo.

El retraso del primer embarazo fue registrado en todos los grupos de edad, pero quienes mencionaron el uso de métodos anticonceptivos para 
hacerlo fueron, en mayor proporción las adolescentes (23.1 por ciento), seguido de las adultas jóvenes (21.8 por ciento) (Tabla 3 ).

Debe precisarse que entre las adolescentes se registraron distintos comportamientos respecto del retraso del primer embarazo según los grupos de edad. Los resultados muestran que el embarazo ocurrió en la totalidad de las mujeres que se unieron antes de cumplir 15 años; mientras que, en las unidas entre los 15 y 16 años, 78.9 por ciento y, entre quienes se unieron entre los 17 y 19 años, solamente una tercera parte ha estado embarazada (33.3 por ciento) (Tabla 4), registrando una relación inversa entre la edad de unión de las adolescentes y el uso de métodos anticonceptivos para posponer el primer embarazo. Una probable explicación es el mayor nivel de escolaridad alcanzado por las adolescentes de mayor edad. En estas últimas 66.7 por ciento tenía secundaria terminada o más, a la vez que la pareja, en 66.7 por ciento había concluido el bachillerato, encontrándose una asociación entre el nivel de escolaridad de la adolescente y el de su pareja en las uniones establecidas entre los 15 y 17 años $(\mathrm{p}<0.000)$, coincidiendo parejas con escolaridad más elevada que el resto de las adolescentes unidas y de otras mujeres en la comunidad.

Tabla 4: Edad a la unión de las adolescentes por condición de embarazo

\begin{tabular}{lrrr}
\hline Edad a la unión & Unidas embarazadas & Unidas no embarazadas & Total \\
& $\mathrm{n}=21$ & $\mathrm{n}=6$ & $\mathrm{n}=27$ \\
& $\%$ & $\%$ & $\%$ \\
\hline $13-14$ & 100.0 & 0.0 & 100.0 \\
$15-16$ & 78.9 & 21.1 & 100.0 \\
$17-19$ & 33.3 & 66.7 & 100.0 \\
Total & 76.9 & 23.1 & 100.0 \\
Media y rango & $15.15(13-17)$ & $16.33(15-19)$ & $15.42(13-19)$ \\
\hline
\end{tabular}

Fuente: elaboración propia con base en datos del trabajo de campo.

El trabajo remunerado de las mujeres es una condición que influencia notablemente la decisión de posponer el primer embarazo y que se suma al efecto de la escolaridad señalado previamente. Todas las adolescentes unidas nunca embarazadas, antes de unirse habían trabajado y obtenido dinero por su trabajo obteniendo entre 300 y 1,000 pesos mensuales antes de unirse y continúan trabajando posterior a la unión: 100 por ciento realiza alfarería artesanal que es la actividad económica mejor remunerada pero 
que les requiere mayor tiempo realizar ${ }^{14}$ (Tabla 5), registrándose diferencias significativas en la ocupación de las adolescentes por condición de embarazo $\left(\mathrm{X}^{2}=10.636,2 \mathrm{gl}, 0.005\right)$, lo que, a su vez evidencia cómo se influyen mutuamente el trabajo productivo y el reproductivo.

Tabla 5: Ocupación en mujeres unidas por condición de embarazo

\begin{tabular}{|c|c|c|c|c|}
\hline Ocupación & $\begin{array}{r}\text { Unidas } \\
\text { embarazadas } \\
\mathrm{n}=20 \\
\end{array}$ & $\begin{array}{r}\text { Unidas nunca } \\
\text { embarazadas } \\
\mathrm{n}=6\end{array}$ & $\begin{array}{r}\text { Total } \\
\mathrm{n}=26\end{array}$ & $\mathrm{X}^{2}$, gl y valor de $\mathrm{p}$ \\
\hline & $\%$ & $\%$ & $\%$ & \\
\hline Hogar & 15.0 & 0.0 & 11.5 & \multirow{4}{*}{$10.636,2 \mathrm{gl}, 0.005$} \\
\hline $\begin{array}{l}\text { Hace piezas } \\
\text { de barro }\end{array}$ & 25.0 & 100.0 & 42.3 & \\
\hline $\begin{array}{l}\text { Borda blusas } \\
\text { de manta }\end{array}$ & 60.0 & 0.0 & 46.2 & \\
\hline Total & 100.0 & 100.0 & 100.0 & \\
\hline
\end{tabular}

Fuente: elaboración propia con base en datos del trabajo de campo.

En esta comunidad el trabajo remunerado es valorado de manera muy positiva por las mujeres y es una actividad que les permite posponer el primer embarazo, lo que, aunado a una mayor escolaridad y por ello, probablemente mayor conocimiento y acceso a los métodos anticonceptivos, ha modificado las expectativas sobre el matrimonio y el deseo de tener hijos o de tenerlos inmediatamente después de la unión.

\section{Algunas expectativas de las mujeres solteras frente al matrimonio y el tener hijos}

Para tener un panorama más amplio del cambio reproductivo en la comunidad, se presentan las expectativas que las mujeres solteras de 15 a 24 años tienen sobre el matrimonio y el tener hijos. De las solteras entrevistadas de 15 a 19 años, $(n=104), 19.7$ por ciento dijeron que no les gustaría casarse ni tener hijos (Tabla 5). Algunos de los comentarios de las mujeres de este grupo de edad respecto a su negativa a unirse fueron: "no me gusta"; "no quiero, no me gusta"; "porque miro que le pegan a mi mamá"; "porque

14 De las mujeres que bordan blusas artesanales, 51 por ciento tienen un ingreso mensual menor a $\$ 500.00$ pesos, a diferencia de quienes hacen piezas de barro para vender, en las que 52.8 por ciento tienen ingresos de $\$ 500.00$ a mil pesos mensuales. El monto del ingreso se relaciona también con el número de horas trabajadas: a mayor número de horas de trabajo, mayores ingresos, dependiendo del tipo de actividad productiva que realicen (bordado o alfarería). Algunas alfareras muy especializadas pueden tener un ingreso de $\$ 20,000.00$ a $\$ 25,000.00$ pesos por una pieza de jaguar. 
quiero trabajar y estudiar; "por no aguantar levantar temprano"; mientras que algunas de las razones para no querer tener hijos fueron: "cuesta para mantenerlos, para criarlos", "no me gustan los niños", "porque hay que levantarse temprano". De ellas, ocho ya habían sido pedidas en matrimonio, pero rechazaron casarse: "claro que no quiero porque aún falta crecer"; "porque no me gustaba (el hombre que la pidió)".

De las 93 solteras de 20 a 24 años, 16.7 por ciento dijeron no querer unirse: "no quiero"; "porque quiero ir a otros lugares"; "porque no sé"; y 12.9 por ciento, no quieren tener hijos: "no quiero"; "porque quitan tiempo". De ellas, tres ya habían sido pedidas y rechazaron la unión: "no quiero responsabilidad todavía, quiero disfrutar mi juventud"; "porque no quise" (Tabla 6).

Tabla 6: Solteras según deseo o no de unirse o de embarazarse y tener hijos

\begin{tabular}{lrrrr}
\hline $\begin{array}{l}\text { Mujeres solteras } \\
\text { de } 15 \text { a } 29 \text { años }\end{array}$ & $\begin{array}{r}\text { Sí } \\
\%\end{array}$ & $\begin{array}{r}\text { No } \\
\%\end{array}$ & $\begin{array}{r}\text { No sabe } \\
\%\end{array}$ & $\begin{array}{r}\text { Total } \\
\%\end{array}$ \\
\hline $\begin{array}{l}\text { ¿Le gustaría o le hubiera } \\
\text { gustado casarse? }\end{array}$ & & & & \\
15 a 19 & 76.4 & 19.7 & 3.9 & 100.0 \\
20 a 24 & 80.7 & 16.1 & 3.2 & 100.0 \\
25 a 29 & 47.9 & 47.8 & 4.3 & 100.0 \\
¿Le gustaría o le hubiera & & & & \\
gustado tener hijos? & & & & \\
15 a 19 & 77.7 & 19.7 & 2.6 & 100.0 \\
20 a 24 & 80.6 & 12.9 & 6.5 & 100.0 \\
25 a 29 & 52.2 & 39.1 & 8.7 & 100.0 \\
\hline
\end{tabular}

Fuente: elaboración propia con base en datos del trabajo de campo.

Esos datos muestran con claridad un porcentaje elevado de rechazo al matrimonio y a tener hijos; las razones que las solteras dieron se relacionan con la sobrecarga de trabajo productivo y reproductivo, por las restricciones en la movilidad que impone la unión y la maternidad, y por la violencia que han observado en otras uniones, así como la probabilidad de abandono de las mujeres, lo cual ocurre en su mayoría cuando ya tienen hijos. El trabajo remunerado que les proporciona mayores ingresos y autonomía mientras están solteras explica en parte el deseo de no casarse y de no tener hijos en una de cada cinco mujeres solteras adolescentes. 
El rechazar el ser pedida fue observado tanto en las adolescentes como en las mujeres adultas jóvenes, lo que demuestra rasgos de autonomía que se manifiestan abiertamente en su capacidad de elección. En este caso, no solamente se refiere a la elección de la pareja o la forma en que establecen la unión, sino a la decisión de no unirse y no tener hijos, lo que hace evidente un cambio reproductivo importante en la comunidad. La proporción de mujeres unidas también ha disminuido en los grupos de menor edad, siendo de 88.4 por ciento en las de 44 a 49 años, 93.1 por ciento en las de 40 a $44,81.3$ por ciento en las de 35 a $39,84.4$ por ciento en las de 30 a $34,67.5$ por ciento en las de 25 a $29,63.4$ por ciento en las de 20 a 24 y de 25.0 por ciento en las mujeres de 15 a 19 años, lo que parece sustenta una reducción en el porcentaje de uniones compatible lo señalado respecto a la decisión de las mujeres de no unirse y, por tanto, de no tener hijos.

\section{Factores explicativos del embarazo adolescente}

Finalmente, se decidió analizar cuantitativamente algunos factores explicativos del embarazo adolescente en las mujeres de 15 a 49 años. Para ello fueron incorporados tres de los elementos descritos hasta ahora: el matrimonio forzado, la escolaridad de las mujeres y el trabajo remunerado. Los resultados de la regresión logística binaria se muestran en la Tabla 7.

Tabla 7: Factores explicativos del embarazo adolescente de las mujeres de 15-49 años

\begin{tabular}{lrrrrr}
\hline & B & E.T. & Wald & Sig. (P) & $\operatorname{Exp(B)}$ \\
\hline Variables independientes & & & & & \\
Trabajo remunerado (si/no) & -0.699 & 0.302 & 5.375 & 0.02 & 0.497 \\
Quería unirse o casarse (si/no) & 1.507 & 0.574 & 6.9 & 0.009 & 4.513 \\
Escolaridad & & & 12.238 & 0.002 & \\
Primaria incompleta & 1.597 & 0.787 & 4.119 & 0.042 & 4.94 \\
Hasta secundaria & 2.198 & 0.794 & 7.671 & 0.006 & 9.01 \\
Constante & -2.332 & 1.02 & 5.224 & 0.022 & 0.097 \\
\hline
\end{tabular}

Fuente: elaboración propia con base en datos del trabajo de campo.

Como puede verse, las tres variables fueron significativas en su relación con la probabilidad de ocurrencia del embarazo adolescente. Notablemente, el matrimonio forzado (no quería casarse), es una variable explicativa importante (Tabla 7); las mujeres que no querían casarse o unirse y fueron forzadas a hacerlo tuvieron 4.5 veces mayor probabilidad de tener un em- 
barazo en la adolescencia. Y ello ocurre pese a la disminución en la edad de unión documentada a lo largo de este texto.

\section{DISCUSIÓN Y CONCLUSIONES}

Los cambios reproductivos en la vida de las mujeres tseltales de Amatenango del Valle, nos llevan a las siguientes reflexiones y conclusiones.

Ante el reconocimiento de la importante participación económica de las mujeres por parte de la comunidad y las familias, la práctica del matrimonio forzado ha disminuido asociado a la participación de las mujeres en actividades productivas remuneradas, mismas que han tenido efecto en dos ámbitos: por una parte, dependen menos económicamente del varón y, para los padres las hijas ya no representan una carga económica, sino que contribuyen al sostenimiento del hogar; esto es diferente a lo reportado por Lagarde (2011), quien, para otros contextos señalaba que las mujeres "a cierta edad, se convierten en un estorbo, deben partir, deben pasar a manos de otro hombre (...) si no lo hacen se vuelve una carga económica, social y afectiva". Este cambio, ha ayudado a que las mujeres en esta comunidad, sobre todo las más jóvenes puedan decidir con quién o simplemente no casarse, aun frente a las presiones de género que ejercen los familiares y la comunidad.

Por otra parte, la escolaridad de las mujeres se ha incrementado significativamente, con registro de ingreso a la educación media y superior de algunas mujeres. Esta condición ha contribuido a la disminución del matrimonio forzado porque les permite retar la autoridad de los padres, unirse "voluntariamente", y retrasar el primer embarazo posterior a la unión mediante el uso de métodos anticonceptivos modernos. El acceso a la educación formal y a las escuelas les abre nuevas posibilidades de relacionarse entre los jóvenes y el contacto con nuevas formas de pensamiento. El incremento de la escolaridad promedio, como producto de la modernización, afecta las prácticas reproductivas en dos sentidos: por una parte, el aumento del grado de escolaridad de las mujeres se ha acompañado de un descenso de la proporción de matrimonios forzados y ha incrementado las uniones "voluntarias"; por otro lado, ha disminuido la edad promedio de unión, a la vez que la proporción de unidas entre los 12 y 19 años es de 16 por ciento, cifra ligeramente superior a la media nacional para asentamientos menores a 15 mil habitantes. Además, tiene un importante papel explicativo en el uso de métodos anticonceptivos para retrasar el primer embarazo, ya que se encuentra asociado a la mayor escolaridad. Así, el embarazo adolescente, sobre todo a edades muy tempranas (12 a 14 años) 
ocurre por matrimonios arreglados, aunque con el consentimiento de la novia, seguido de embarazo, y se concentra, por la edad de las mujeres, en el grupo con menor escolaridad.

Los servicios de salud y las campañas de planificación familiar han tenido un efecto muy importante en la vida reproductiva de las mujeres indígenas tseltales de Amatenango del Valle, pero vinculado a la mayor escolaridad de las mujeres unidas. El trabajo remunerado y el acceso a la educación de las mujeres, ha permitido que las adolescentes y mujeres se enfrenten a las normas tradicionales de género, "rechazando algunas, actualizando otras u otorgándoles otros sentidos" (Reartes, 2011: 90) que, en el caso de esta comunidad, implica el rechazo al matrimonio, el retraso del primer embarazo o la construcción de alternativas de vida diferentes a la de madres y esposas. Las normas comunitarias de género se han tensionado frente a los nuevos conocimientos y experiencias de las mujeres; se podría decir que, sobre todo las de mayor escolaridad, tienen mayor dominio y control sobre su propia vida. El trabajo remunerado les ha permitido a muchas de ellas a acceder y manejar sus propios recursos materiales y económicos (Comisión de Mujeres y Desarrollo, 2007), lo que les ha permitido resistir las rígidas normas de género y la violencia ejercida contra ellas por los hombres, para mejorar sus condiciones de vida expresadas en su mayor autonomía en la toma de decisiones económicas y en las prácticas reproductivas para disminuir o evitar la sobrecarga de trabajo y la exposición a la violencia por parte de su pareja; esto puede considerarse un logro en una comunidad donde la violencia contra las mujeres es generalizada y podría ser interpretada como un indicador de mejora en las relaciones de género e igualdad entre hombres y mujeres en la comunidad. No obstante, los resultados de este estudio muestran que los varones no han cambiado la manera de relacionarse con las mujeres; son ellas, quienes, aun a costa de no unirse o no ser madres, resisten dicha violencia y transgreden - no sin consecuencias - , las normas que exigen ser "pedidas" para contraer matrimonio o unirse, registrándose en las actuales adolescentes unidas, que en todos los casos de la unión fue "voluntaria".

Debido a la permanencia de las desiguales relaciones de género, la prohibición legal del matrimonio infantil no ayudaría a reducir las uniones tempranas. De hecho, en una comunidad como esta en la que ha disminuido la edad de unión, y persiste la norma que legitima los matrimonios arreglados en niñas, se ha observado más recientemente, la ocurrencia de uniones fuera de la norma (no esperar a ser pedidas) y el no establecimiento de una unión formal (matrimonio legal), lo que coloca a las menores 
en condición de alta vulnerabilidad; si a ello se agrega el cambio en la legislación, el resultado podría ser un incremento de la vulnerabilidad de las menores y mayor inestabilidad de las uniones. Como se documentó en este trabajo, las y los jóvenes se unen "voluntariamente", particularmente entre quienes tienen mayor escolaridad, mencionando haberse enamorado o que su pareja les gustó; sin embargo, el mayor porcentaje de las uniones tempranas no se concreta de manera formal o legal, sino en uniones libres y la tasa de abandono o separación es muy elevada, mucho más que la registrada por mujeres de mayor edad. Esto muestra la vigencia de las normas de género y el control social al que se enfrentan las mujeres en esta comunidad, lo que podría empeorar con la prohibición del matrimonio de menores de edad.

Se sugiere instrumentar políticas públicas que protejan a las mujeres en general que son víctimas de cualquier tipo de violencia, principalmente a las mujeres que se unen a temprana edad, porque tienen "mayor riesgo de sufrir violencia doméstica y violencia sexual de sus parejas" (ONU MUJERES, 2016: 8; Casique y Castro, 2018); en el caso de esta comunidad, porque son uniones más inestables y siempre acompañadas de embarazos, dejándolas, en el caso de separarse o ser abandonadas, en una condición de elevada vulnerabilidad.

Finalmente, para disminuir las prácticas de uniones tempranas y embarazos adolescentes, se debe pensar en políticas integrales que consideren las condiciones contextuales de cada grupo social y pueblo originario. Es importante apoyar las actividades productivas y económicas de las adolescentes y jóvenes en el medio rural e indígena, crear condiciones de ocupaciones remuneradas (creación de cooperativas y microempresas, y motivarlas a que asistan a la escuela y continúen sus estudios ${ }^{15}$ ) a la par de la mejora de las oportunidades educativas, que, como se ha documentado en este trabajo, tienen un papel clave en el cambio de expectativas y resistencia de las mujeres al matrimonio forzado y la maternidad temprana en un entorno de violencia por la pareja. Aunado a ello, debe realizarse un trabajo permanente, con hombres y mujeres, para el logro de la equidad de género, el respeto a los derechos humanos de las mujeres de los pueblos originarios, así como de sus derechos sexuales y reproductivos, mejorando e incrementando la atención a la salud en las adolescentes, con énfasis en la educación sexual y reproductiva, que, en algunas comunidades como la de este estudio, existía pero ha dejado de funcionar. Sin estas acciones, no

\footnotetext{
15 A veces, muchas de ellas desean continuar estudiando y no pueden porque tienen que contribuir a la economía familiar, por lo que el trabajo infantil puede ser causa de deserción escolar en las poblaciones rurales e indígenas (Pedrero, 2002).
} 
se podrá erradicar la práctica de matrimonios arreglados en niñas, disminuir las uniones tempranas "voluntarias" y el embarazo en adolescentes, o mejorar la calidad de vida de las adolescentes y de las mujeres en general.

\section{REFERENCIAS BIBLIOGRÁFICAS}

Agarwal, B., 1997, Bargaining' and gender relations: within and beyond the household, en FCND Discussion Papers No. 27, U.S.A., Washington, D.C. Food Consumption and Nutrition Division. International Food Policy Research Institute.

Araiza, A., 2006, "Las mujeres indígenas en México: un análisis desde la perspectiva de género", en Quaderns-e de l'Institut Català d'Antropologia, 08(b), 1-17, fecha de consulta 9/11/2018. Disponible en https://www.raco.cat/index.php/QuadernseICA/article/view/56017

Arias, S., Gonzales, P. y Torres, M., 2008, “Consideraciones teóricas y empíricas acerca de la fecundidad adolescente", en Revista de la Facultad de Ciencias Económicas: Investigación y Reflexión, XVI (2), 47-59, fecha de consulta, 20/04/2017. Disponible en https://www.redalyc.org/articulo.oa?id=90916204

Bourdieu, P., 2007, El sentido práctico, siglo XXI Editores, Argentina.

Cámara de Diputados, 2019, Cámara de Diputados aprueba reformas para prohibir el matrimonio infantil, H. Congreso de la Unión, LXIV Legislatura fecha de consulta 3/05/2019. Disponible en http://www5.diputados.gob.mx/index.php/esl/ Comunicacion/Boletines/2019/Abril/30/1584-Camara-de-Diputados-aprueba-reformas-para-prohibir-el-matrimonio-infantil

Casique, I. y Castro, R., 2018, "Magnitudes y determinantes de la violencia ejercida por la pareja en contra de las mujeres. Un análisis comparativo", en Casique, I. y Castro, R. (coords.), Expresiones y contextos de la violencia contra las mujeres en México, pp. 183-255, Instituto Nacional de las Mujeres, Centro Regional de Investigaciones Multidisciplinarias, México.

Chantler, K., 2012, "Recognition of and intervention in forced marriage as a form of violence and abuse", en Trauma, Violence and Abuse, 13(3), 176-83.

Collier, G., 1992, "Busqueda de alimento y busqueda de dinero: Cambios en las relaciones de producción en Zinacantán, Chiapas”, en Appendini, (Ed.), Reestruccturación económica y subsistencia rural: El maíz y la crisisi de los ochenta, pp. 53-83, Colegio de México, México.

Consejo Nacional de Evaluación de la Politica de Desarrollo Social, 2010, Porcentaje de población en situación de pobreza 2010, Chiapas, fecha de consulta, 30/01/2017. Disponible en http://www.coneval.org.mx/coordinacion/entidades/ Chiapas/Paginas/pob_municipal.aspx

Comisión de Mujeres y Desarrollo, 2007, El proceso de empoderamiento de las mujeres: guía metodológica, fecha de consulta, 10/05/2019. Disponible en http:// www.dhl.hegoa.ehu.es/ficheros/0000/0251/proceso_empoderamiento_mujeres CFD.pdf 
Chávez, M., Hernández, H., Menkes, C. y Gonzales, A., 2007, “Tendencias de la fecundidad indígena en México, 1997 y 2003”, en Hernández, H. (Ed.). Los indios de México en el siglo XXI, pp. 43-60, Universidad Nacional Autónoma de México y Centro Regional de Investigaciones multidisciplinarias, México.

De Jesús, D., 2011, Adolescencias escindidas: sexualidad y reproducción adolescente en contextos urbano-marginales de Nuevo León, Universidad Autónoma de Nuevo León, México, fecha de consulta, 20/05/2017. Disponible en http://eprints. uanl.mx/2374/1/Libro_adolescencias_escindidas.pdf

El Universal, 2019, "Chiapas, primer lugar nacional en matrimonios infantiles", fecha de consulta, 5/05/2018. Disponible en https://www.yucatan.com.mx/mexico/chiapas-primer-lugar-nacional-en-matrimonios-infantiles

Frías, S., 2017, "Family and parther violence against women: forced marriage in mexican indigenous comunities", en International Journal of Law, Policy and the Family, 31(1), 60-78, fecha de consulta 30/04/2018. Disponible en https://academic.oup.com/lawfam/article-abstract/31/1/60/3065578?redirectedFrom=fulltext

Fondo de Población de las Naciones Unidas, 2013, Maternidad en la niñez: enfrentar en reto del embarazo en adolescentes, fecha de consulta, 20 de octubre 2017. Disponible en https://www.unfpa.org/sites/default/files/pub-pdf/ES-SWOP2013. pdf

Fondo de Población de las Naciones Unidas, 2012, Marrying too Young end child marriage, fecha de consulta, 30/04/2019. Disponible en https://www.unfpa.org/ end-child-marriage

Freyermuth, G., Arguello, H. y Zarco, Á., 2014, Monitoreo de la atención de la salud sexual y reproductiva en adolescentes indigenas, CIESAS/INMUJERES, México.

Frulli, M., 2008, “Advancing International criminal law. The special court for Sierra Leone recognizes forced marriage as a'New'Crime against Humanity", en Journal of International Criminal Justice 6(5), 1033-1042, fecha de consulta, 30/04/2018. Disponible en https://papers.ssrn.com/sol3/papers.cfm?abstract $\mathrm{id}=2014731$

Gonzáles, S. y Mojarro, M., 2011, “Algunas dimensiones de la nupcialidad y la situación de las mujeres en ocho regiones indígenas de México", en Vázquez, G. y Reyna, E. (Ed.), Retos, problemáticas y políticas de la población indígena en México, (pp. 181-202), Miguel Ángel Porrúa, Universidad Autónoma del Estado de Hidalgo, México.

Gonzáles, S., 1999, “Las 'costumbres' de matrimonio en el México indígena contemporáneo", en Figueroa, B. (editor), México diverso y desigual: Enfoques sociodemográficos, (pp. 87-105), El Colegio de México, Sociedad Mexicana de Demografía, México.

Igareda, N., 2015, "El problema de los matrimonios forzados como violencia de género", en Oñati Socio-legal Series, 2(5), 613-624, fecha de consulta, 3/12/2018. Disponible en http://ssrn.com/abstract=2611913 
Instituto Nacional de Estadística y Geografía, 2015, Encuesta Intercensal 2015. Microdatos, fecha de consulta, 20/04/2018. Disponible en https://www.inegi.org. $\mathrm{mx} /$ programas/intercensal/2015/default.html\#Microdatos

Instituto Nacional de Estadística y Geografía, 2016, Anuario estadístico y geográfico de Chiapas 2016, fecha de consulta, 20/04/2017. Disponible en https://www. datatur.sectur.gob.mx/ITxEF_Docs/CHIS_ANUARIO_PDF.pdf

Instituto Nacional de las Mujeres, $\mathrm{s} / \mathrm{f}$, "Sistema de indicadores de género: madres adolescentes", fecha de consulta, 30/04/2019. Disponible en http://estadistica.inmujeres.gob.mx/formas/tarjetas/Madres_adolescentes1.pdf

Kopelman, M., 2016, "The forced marriage of minors: a neglected form of child abuse", en The Journal of Law, Medicine \& Ethic, 44(1), 173-81, fecha de consulta, 30/04/2018. Doi: 10.1177/1073110516644208

Lagarde, M., 2011, Los cautiverios de las mujeres: madresposas, monjas, putas, presas y locas, Universidad Nacional Autónoma de México, México.

Linares, B., 2018, La no maternidad en México: mujeres indígenas de Amatenango del Valle que no son madres, tesis de doctorado, El Colegio de la Frontera Sur, San Cristóbal de Las Casas, Chiapas.

Machel, G., Pires, E., Carlsson, G., 2013, "The world we want: an end child marriage", en The Lancet, 382(9897), 1005-1006, fecha de consulta, 30/04/2018. DOI: https://doi.org/10.1016/S0140-6736(13)61944-3

Menkes, C. y Stern, C., 2012, "Embarazo adolescente y estratificación social”, en Stern, C. (coords.), El "problema" del embarazo en la adolescencia: contribuciones a un debate, (pp. 227-269), El Colegio de México, México.

Menkes, C. y Suárez, L., 2003, "Sexualidad y embarazo adolescente en México", en Papeles de Población, 9(35), 1-31, fecha de consulta, 20/04/2017. Disponible en https://www.redalyc.org/pdf/112/11203511.pdf

Menkes, C. y Suárez, L., 2013, “El embarazo de los adolescentes en México: ¿es deseado?", en Coyuntura Demográfica, 4(0), 21-28, fecha de consulta, 20/04/2018. Disponible en http://www.somede.org/images/dc4/2elembarazo.pdf

Mindek, D., 2003, "Formación y disolución del matrimonio indígena: una revisión crítica”, en Robichaux, D., (Comp.), El matrimonio en mesoamérica ayer y hoy, (pp. 331-360), Universidad Iberoamericana, México.

Mojarro, M., Gonzáles, S. y Valdez, R., 2008, "Salud y derechos reproductivos de las mujeres entrevistadas, conclusiones y reflexiones finales", en Encuesta de Salud y Derechos de las Mujeres Indigenas (ENSADEMI 2008), (pp. 61-69; 117 122), Instituto Nacional de Salud Pública, México.

Nash, J., 1993, "Maya household production in the Word market. The potters of Amatenango, del Valle, Chiapas, México", en Crasfs in the world market: The impacto of global exchange on middle american artisans (pp. 127-153). State University of New York Press, Albany. 
Torres, N., 2015, "Matrimonio forzado: aproximación fenomenológica y análisis de los procesos de incriminación" en Estudios Penales y Criminológicos, (XXXV), 831-917, fecha de consulta, 20 de octubre 2018. Disponible en http:// www.usc.es/revistas/index.php/epc/article/view/2703/3269

Ojeda, N., 1999, "Porpuesta teórica y metodologica para estudiar la determinación social de la conceptualización de la salud reproductiva de las mujeres", en Ojeda, N., (Coords.), Género, familia y conceptualización de la salud reproductiva en México, (pp. 21-65), El Colegio de la Frontera Norte, México.

Organización de las Naciones Unidas para la Infancia, 2014, Informe de la Oficina del Alto Comisionado de las Naciones Unidas para los Derechos Humanos. Prevención y eliminación del matrimonio infantil, precoz y forzado, fecha de consulta, 20 de abril 2018. Disponible en https://www.acnur.org/fileadmin/Documentos/ BDL/2014/9585.pdf

Organización de las Naciones Unidas, Mujeres, México (ONU MUJERES), 2016, "Matrimonio de niñas y uniones tempranas", en Serie: Transformando nuestro mundo, fecha de consulta, 20 de abril 2017. Disponible en http://www.legisver. gob.mx/equidadNotas/publicacionLXIII/MATRIMONIO\%20INFANTIL_.pdf

Organización Mundial de la Salud, 2012, Prevenir el embarazo precoz y los resultados reproductivos adversos en adolescentes en los países en desarrollo: las evidencias, fecha de consulta, 30 de abril 2018. Disponible en https://apps.who. int/iris/handle/10665/78253

Pedrero, M., 2002, “Empleo en zonas indígenas”, en Papeles de población, 8(31), 117-162, fecha de consulta 10 de mayo de 2019. Disponible en http://www.scielo.org.mx/scielo.php?script=sci_arttext\&pid=S1405-74252002000100006\&ln$\mathrm{g}=$ es\&tlng=es.

Pozas, R., 2012, "La organización social”, en Pozas, R. (editor), Chamula. Un pueblo indio de los Altos de Chiapas, (pp. 25-168), Comisión Nacional para el Desarrollo de los Pueblos Indios, México.

Ramírez, D., Nazar, A., Mariaca., R. y Olivera, M., 2005, “Género y negociación reproductiva en una comunidad rural de Chiapas", en Revista Mexicana de Sociología, 67(4), 687-727.

Ramos, D., 2003, Capitales locales en procesos globales: el caso de las transformaciones productivas en Amatenango del Valle, Chiapas (Tesis Doctoral), El Colegio de la Frontera Sur, San Cristóbal de Las Casas, Chiapas.

Reartes, D., 2011, “Género, etnia y generación en la prevención e interrupción de embarazos en jóvenes estudiantes hablantes de lenguas mayas migrantes a San Cristóbal de las Casas, Chiapas (México)", en Cuaderno de Antropología Social, (33), 71-92, fecha de consulta, 20 de abril 2018. Disponible en http://revistascientificas.filo.uba.ar/index.php/CAS/article/view/1418/1359

Sen, A., 1990, "Gender and cooperative conflicts", en Tinker, I. (coomp.), Persistent inequalities. Women and world development, Nueva York, Oxford University Press. 
Simmons, F., y Burn, J., 2013, "Without consent: forced marriage in Australia", en Melbourne University Law Review, 36(6), 970-1008, fecha de consulta, 30 de abril 2018. Disponible en http://www.austlii.edu.au/au/journals/MelbULawRw/2012/24.html

Solís, P., 2013, “Las nuevas uniones libres en México: más tempranas e inestables, pero tan fecundas como los matrimonios", en Coyuntura Demográfica, 4(0), 3136, fecha de consulta, 20 de abril 2018. Disponible en http://www.somede.org/ images/dc4/3lasnuevas.pdf

Stern, C., 2004, "Vulnerabilidad social y embarazo adolescente en México", en Papeles de Población, 10(39), 129-158, fecha de consulta, 20 de abril 2018. Disponible en https://www.redalyc.org/articulo.oa?id=11203906

Scott, J., Hacker, M., Averbacha, S., Modesta, A., Cornishe, S., Spencere, D., Murphye, M., Parmarc, P., 2014, "Influences of sex, age and education on attitudes towards gender inequitable norms and practices in South Sudan", en Global Public Health, 9(7), 773-786, fecha de consulta, 30 de abril 2018. Disponible en http:// dx.doi.org/10.1080/17441692.2014.928347

Valdez-Santiago, R., Ávila-Burgos, L., Hidalgo-Solórzano, E., Palma, O., 2008, "Metodologías de la ENSADEMI", en Encuesta de Salud y Derechos Humanos de las Indígenas (ENSADEMI), Instituto Nacional de Salud Pública, (29-41), México.

Warria, A., 2017, "Forced child marriages as a form of child trafficking", en Children and Youth Services Review, (79), 274-279, fecha de consulta, 30 de abril 2018. Disponible en https://www.sciencedirect.com/science/ar

ticle/pii/S0190740916303929\#!

\section{RESUMEN CURRICULAR DE LAS AUTORAS}

Juana Luna Pérez

Licenciada en Gestión y Autodesarrollo Indígena por la Universidad Autónoma de Chiapas y Maestra en Ciencias de Recursos Naturales y Desarrollo Rural con Orientación en Salud, Equidad y Sustentabilidad de El Colegio de la Frontera Sur.

Dirección electrónica: juanitaluna8502@gmail.com

\section{Austreberta Nazar Beutelspacher}

Médica Cirujana por la Universidad Autónoma de Chiapas, Maestra en Medicina Social por la Universidad Autónoma Metropolitana-Xochimilco, Especialista en Epidemiología Aplicada por la Secretaría de Salud y los Centros para el Control de Enfermedades de los Estados Unidos, Doctora en Estudios del Desarrollo Rural por el Colegio de Postgraduados. Desde 1992 labora como investigadora en el Departamento de Salud de El Cole- 
gio de la Frontera Sur en Chiapas. Ha sido Directora del Área Sociedad, Cultura y Salud, y Directora de Posgrado de El Colegio de la Frontera Sur. Es miembro del Sistema Nacional de Investigadores nivel III, y miembro regular de la Academia Mexicana de Ciencias. Ha sido consultora del Banco Interamericano de Desarrollo. Sus áreas de interés son: Salud, género y desigualdad social. Entre sus publicaciones destacan: “ ¿Does contraception benefit women? Structure, Agency and Well-being in rural México", en B. Agarwal, J. Humpries and I. Robeyns (Ed). Capabilities, Freedom, and Equality. Amartya Sen's Work from a Gender Perspective. Oxford University Press; "Education and Non-Use of Contraceptives among Poor Women in Chiapas, Mexico", in International Family Planning Perspectives.

Dirección electrónica: anazar@ecosur.mx

\section{Ramón Mariaca Méndez}

Maestro en Ciencias en Botánica y orientación Etnobotánica y Doctor en Antropología Social. Nivel 2 del Sistema Nacional de Investigadores. Ha sido Presidente de la Asociación Etnobiológica Mexicana, de la Sociedad Latinoamericana de Etnobiología y actualmente de la Sociedad Agroecológica Mexicana. Sus áreas de interés giran en torno a la Etnobiología, la Agroecología y la concepción y aprovechamiento de recursos naturales por grupos mayenses (mayas yucatecos, tsotsiles, tseltales, ch'oles, lacandones, chontales) incluyendo su agricultura tradicional con énfasis en el estudio de la cultura que la involucra, su tecnología, sus instrumentos de trabajo y su agrobiodiversidad (milpa bajo r-t-q, huertos familiares, marceño).

Dirección electrónica: rmariaca@ecosur.mx

\section{Dulce Karol Ramírez López}

Maestra en Ciencias en Recursos Naturales y Desarrollo Rural con orientación en Salud y Desarrollo Comunitario por El Colegio de la Frontera Sur (ECOSUR) y Doctora en Ciencias con orientación en Población, Ambiente y Desarrollo Rural por El Colegio de la Frontera Sur (ECOSUR). Ha sido Catedrática CONACYT, comisionada al Centro de Estudios Superiores de México y Centroamérica de la Universidad de Ciencias y Artes de Chiapas, 2014 a 2018. Actualmente es Profesora investigadora de la Licenciatura en Ciencias Políticas y Administración Pública de la Universidad de Ciencias y Artes de Chiapas (UNICACH). Es miembro del Sistema Nacional de Investigadores del CONACYT e Investigadora Honorífica del Sistema Estatal de Investigadores del Estado de Chiapas. Entre sus publicaciones más 
Matrimonio forzado y embarazo adolescente en indígenas en Amatenango del Valle, Chiapas ... / J. LUNA PÉREZ et al.

importantes destacan: Los rostros de la desigualdad educativa. Racismo y Sexismo en Educación Superior (en coautoría con Flor M. Bermúdez) y, Mujeres migrantes en la frontera sur de México. Aproximaciones desde la interseccionalidad.

Dirección electrónica: dulce.ramirez@unicach.mx

Artículo recibido el 28 de mayo de 2019 y aprobado el 28 de noviembre de 2020. 